\title{
Late pregnancy ultrasound to screen for and manage potential birth complications in nulliparous women: a cost-effectiveness and value of information analysis
}

Edward C.F. Wilson ${ }^{1,2}(0000-0002-8369-1577)$, David Wastlund ${ }^{2,3}(0000-0002-5074-4740)$, Alexandros A. Moraitis ${ }^{4}(0000-0003-4634-1129)$, Gordon C.S. Smith ${ }^{4}(0000-0003-2124-0997)$

${ }^{1}$ Health Economics Group, Norwich Medical School, University of East Anglia, Norwich, NR4 7TJ, UK

${ }^{2}$ The Primary Care Unit, Department of Public Health and Primary Care, University of Cambridge, Cambridge, CB2 OSR, UK

${ }^{3}$ Parexel Access Consulting, Parexel International, Stockholm 103 59, Sweden.

${ }^{4}$ Department of Obstetrics and Gynaecology, University of Cambridge, NIHR Cambridge Biomedical Research Centre, Cambridge, CB2 2SW, UK

Correspondence to:

Edward C.F. Wilson, PhD, Health Economics Group, Norwich Medical School, University of East Anglia, Norwich, NR4 7TJ, UK.

Tel: +44 (0) 1603 593620; E-mail: Ed.Wilson@uea.ac.uk 


\section{Acknowledgements}

This study was funded by the National Institute for Health Research (NIHR) Health Technology Assessment programme, grant number 15/105/01. The funders had no role in study design, data collection and analysis, decision to publish, or preparation of the manuscript. The views expressed here are those of the authors and not necessarily those of the NHS, the NIHR or the Department of Health. The authors wish to thank Alex Heazell for comments on the manuscript. Gordon Smith reports grants and personal fees from GlaxoSmithKline Research and Development Ltd, grants from Sera Prognostics Inc, non-financial support from Illumina Inc, and personal fees from Roche Diagnostics Ltd, outside the submitted work. In addition, Gordon Smith is a named co-inventor in a patent for a novel predictive test for fetal growth restriction pending.

\section{Concise description:}

Late-pregnancy screening is unlikely to be cost-effective, but a rapid presentation-only scan may be.

The evidence is uncertain but an RCT is not warranted.

\section{Highlights:}

- Foetal growth restriction is a major risk factor for stillbirth. A routine late pregnancy ultrasound scan could detect this, as well as other anomalies such as breech presentation.

- Evidence of effect is scarce, but an RCT powered to detect stillbirth would be extremely large and expensive. It is wise to predict the return on investment from research to ensure the maximum benefit from finite resources.

- Current evidence suggests universal late pregnancy ultrasound scans would not be cost-effective in the UK setting. However, a rapid 'presentation-only' scan may be. An RCT powered to detect stillbirth would not be a value for money investment. 


\section{Abstract}

Background: Foetal growth restriction (FGR) is a major risk factor for stillbirth. A routine latepregnancy ultrasound scan could help detect this allowing intervention to reduce the risk of stillbirth. Such a scan could also detect foetal presentation and predict macrosomia. A trial powered to detect stillbirth differences would be extremely large and expensive. It is therefore critical to know whether this would be a good investment of public research funds.

Objective: To estimate the cost-effectiveness of various late-pregnancy screening and management strategies based on current information, and predict the return on investment from further research.

Methods: Synthesis of current evidence structured into a decision model reporting expected costs, QALYs and net benefit over 20 years and value-of-information analysis reporting predicted return on investment from future clinical trials.

Results: Given a willingness to pay of $£ 20,000$ per QALY gained, the most cost-effective strategy is a routine presentation-only scan for all women. Universal ultrasound screening for foetal size is unlikely to be cost-effective. Research exploring the cost implications of induction of labour has the greatest predicted return on investment. A randomised controlled trial with an endpoint of stillbirth is extremely unlikely to be a value for money investment.

Conclusion: Given current UK value-for-money thresholds, the most cost-effective strategy is to offer all pregnant women a presentation-only scan in late pregnancy. A randomised controlled trial of screening and intervention to reduce the risk of stillbirth following universal ultrasound to detect macrosomia or FGR is unlikely to represent a value for money investment. 


\section{Introduction}

Complications of pregnancy, both to mother and baby, are a major determinant of the Global Burden of Disease. ${ }^{1}$ Stillbirth, defined as the baby born dead at 24 weeks gestational age or later, is a major contributor to this: there were a total of 2689 stillbirths in England and Wales in 2018, equating to approximately $0.4 \%$ of all births. ${ }^{2}$ Foetal growth restriction (FGR) is where the baby fails to achieve its genetically determined growth potential, and is a major risk factor for stillbirth. ${ }^{3}$ It is possible that offering a routine ultrasound scan to every mother in late pregnancy (around 36 weeks gestational age) could help detect FGR, allowing intervention to reduce the risk of stillbirth. Furthermore, an ultrasound scan has the potential to detect other conditions which place the pregnancy at risk such as macrosomia (birthweight $>4 \mathrm{~kg}$ ) and foetal presentation (cephalic or breech).

Under current guidelines in England and the rest of the UK, ${ }^{45}$ an ultrasound scan after 28 weeks is offered only where clinically indicated, e.g. relevant medical history, or concerns following clinical examination. An alternative approach is to offer an ultrasound scan to all late-stage pregnancies. This would be expected to identify more pregnancies in need of intervention. However, this could also increase false positive diagnoses leading to unnecessary, and possibly harmful, intervention. The overall balance of risk to harm to foetal health, and whether such a screening programme would represent the best use of health care resources is unknown, and the need to evaluate this has been highlighted previously. ${ }^{6-8}$

A Cochrane review (searching to August 2014) of routine ultrasound in late-stage pregnancy concluded that there was insufficient evidence to recommend universal screening. ${ }^{9}$ However, none of the 13 trials studied screening followed by an intervention, the different trials applied different definitions of screen positive and performed assessments at different gestational ages, and even the meta-analysis was underpowered for plausible estimates of diagnostic and interventional effectiveness. ${ }^{10}$ 
The key pieces of information that can be obtained from a scan around 36 weeks are whether the fetus measures small or large for gestational age (SGA or LGA, defined as foetal size in the $1^{\text {st }}$ or $10^{\text {th }}$ decile of the distribution respectively), and whether the fetus is in a cephalic (head down) presentation. An SGA fetus may be suffering FGR and hence be at increased risk of stillbirth, whilst an LGA fetus may be macrosomic at delivery (defined as birthweight over $4 \mathrm{~kg}$ ), which increases the risk of complications during delivery. We previously reported analyses of a Level 1 study of diagnostic effectiveness ${ }^{11}$ (where the results of the ultrasound scan were blinded) in relation to extremes of fetal size, ${ }^{12} 13$ and we have also reported that, in the same cohort study, a late pregnancy scan identified about $2.5 \%$ of women with a previously undiagnosed breech presentation at 36 weeks. ${ }^{14}$ Our previous work has also estimated the cost-effectiveness scanning for each of these individually, concluding that scanning for $\mathrm{LGA}^{15}$ and $\mathrm{SGA}^{16}$ is unlikely to be worthwhile. However, we predict that a presentation scan could prevent around eight perinatal deaths per annum, and could be cost-neutral to the English National Health Service (NHS) if able to be performed by a midwife as part of a routine antenatal appointment. $^{14}$

In this paper we build on this work, comparing all screening and management strategies simultaneously within one decision model framework. Critically, we use our framework to estimate overall decision uncertainty and perform a value of information analysis ${ }^{17-19}$ to determine whether there is sufficient evidence to make a policy recommendation or whether investment in further research, for example a randomised controlled trial or other data gathering exercise, would represent value for money for a major public sector funder of research (the National Institute for Health Research, England, UK). This is of particular importance, given that most existing studies (and systematic review ${ }^{20}$ ) were underpowered to detect a statistically significant difference in stillbirth rates between routine and selective screening arms. A new and sufficiently powered clinical trial would need to be extremely large, and thus expensive. It is vital, therefore, to consider whether this is the best use of scarce public funds, or whether more health could be generated for the population from investment in other studies, or direct patient care. 


\section{Methods}

\section{Population}

The target population is singleton nulliparous pregnancies (i.e. babies born to new mothers), in England.

\section{Comparator strategies}

The comparator strategies comprise both a screening option and subsequent management. Screening options are 'selective', 'universal breech' and 'universal'. All scans are assumed to take place at between 36 weeks and 36 weeks +6 days gestational age. 'Selective' screening means only those mothers who are clinically indicated for a late pregnancy scan receive one, assumed to reflect the status quo. ${ }^{45}$ The 'universal breech' scanning strategy offers all mothers a simple presentation-only scan, i.e. solely to determine the orientation of the foetus. It is assumed performed by a midwife using a point of care ultrasound device as part of a routine antenatal contact. 'Universal' screening is defined as all mothers receiving an ultrasound scan incorporating measurements to estimate foetal size. Given the simplicity of establishing foetal presentation, this scan would also identify any babies in the breech position. Findings from a presentation scan can be either cephalic or breech, and foetal size could be either appropriate, small or large for gestational age (AGA, SGA and LGA, respectively).

If a breech presentation is identified, all mothers are assumed offered external cephalic version (ECV, manual manipulation of the mother's belly to turn the foetus to a cephalic presentation), unless contraindicated. If this is declined or unsuccessful, an elective Caesarean section may be scheduled. If LGA is detected, the mother may be offered either induction of labour (loL) or expectant management. If SGA is detected, all mothers are offered induction of labour.

We therefore compare six alternative screening and management policies comprising three possible screening modes and two alternative management plans, numbered 1-6 and summarised in Table 1. 


\section{Model structure}

The model structure is a decision tree with four sections covering breech, LGA, SGA and AGA (Figure 1 and Appendix 1). It was established by discussion amongst the study team, comprising economists and clinicians. For parsimony, we assume they are all mutually exclusive. This is logically true for LGA, SGA and AGA, but a baby may be both breech and LGA, for example. The structure is arranged hierarchically, with breech position first, as this is most easily and reliably identified.

We assume a presentation-only scan is perfectly predictive of breech (i.e. $100 \%$ sensitive and specific). However, our model allows for false negatives which are interpreted as undetected breech deliveries under the selective scanning strategy (node B_B, Figure 1). Where breech is detected, ECV is offered which may be successful or not. If unsuccessful, an elective Caesarean section may be scheduled. In either case, the baby may spontaneously revert to breech or cephalic position. Reversion to breech can lead to a vaginal breech delivery or emergency Caesarean section. Outcomes from delivery comprise none, moderate or severe morbidity, or stillbirth. Surviving infants could subsequently have no long-term complications, special educational needs, severe neurological morbidity or neonatal/infant mortality. The risk of long-term complications increases with neonatal morbidity severity (Appendix 1, Figure A1.1).

An LGA baby may or may not be diagnosed as such, determined by the sensitivity of the scan (Figure 1, node L_B). A positive scan can be managed either with induction of labour or expectant management, determined by the overall strategy (Table 1, Figure 1, node MGT_LGA_TP). Induction is assumed to reduce the risk of emergency Caesarean section (Figure 1, nodes L_C3 and L_C2 respectively). Delivery of a macrosomic baby leads to either no complications or respiratory morbidity, shoulder dystocia (trapping of the shoulder behind the mother's pubic bone) with attendant risk of transient or permanent brachial plexus injury (damage to the nerves of the arm) and acidosis (lowered blood pH usually due to build-up of carbon dioxide), other acidosis (i.e. not related to shoulder dystocia) or neonatal mortality (Appendix 1, Figure A1.2). Long term complications are 
divided into none, special educational needs, severe neurological morbidity and neonatal/infant mortality (mirroring the structure of the breech arms, Appendix 1, Figure A1.1).

An SGA baby diagnosed as such will undergo induction of labour, with either a vaginal or emergency Caesarean section as the delivery mode. Undetected SGA babies are not induced and undergo either vaginal or emergency Caesarean section, with differing probabilities (Figure 1, nodes S_B, S_C3 and S_C2 respectively). Infants are then at risk of none, moderate or severe morbidity or stillbirth, with long term outcomes comprising no complications, special educational needs, severe neurological morbidity and neonatal/infant mortality (Appendix 1, Figure A1.3), mirroring the structure of the breech arms (Appendix 1, Figure A1.1).

AGA babies may be falsely diagnosed as SGA or LGA, in which case the management and patient pathways are as per the true positives described above (Figure 1, node B). However, the risks of adverse outcomes vary as described below ('model data'). Babies correctly identified as AGA undergo routine deliveries, with a 'background' risk of conversion to emergency Caesarean section for reasons other than foetal size or presentation (Figure 1, node C1). The expanded tree for AGA babies is shown in Appendix 1, Figure A1.4.

\section{Model data}

Data to populate the model were extracted from multiple sources in the literature $\mathrm{e}^{12-1421-63}$ (Appendix 2, Table A2.1). Good quality systematic reviews and meta-analyses were prioritised, followed by large, good quality clinical trials or cohort studies as appropriate. Where possible, probabilities were expressed as a baseline and odds ratio (or relative risk where odds were not calculable). Unit costs pertained to a $2016 / 17$ price year. Care was taken to appropriately reflect uncertainty in all parameters, as specified in the assigned probability distributions (Appendix 2, Table A2.1). Where no evidence for a parameter existed, we relied on expert opinion either to judge whether a study in a related area provided a sufficient proxy, or to provide a central estimate and credible interval representing beliefs about plausible values for the parameter. Source data for parameters were 
assigned a subjective quality rating, high representing a source of directly relevant data, and low representing use of indirectly relevant or no data, revised with expert opinion. Model inputs and details of derivation are reported in Appendix 2.

\section{Analysis}

The model was analysed via Monte Carlo simulation, with the appropriate number of simulations determined by the trade-off between minimising Monte Carlo error and computational expense (Appendix 3). Model outcomes comprised mean, variance and covariance of costs and qualityadjusted life years (QALYs), reported as mean and 95\% credibility intervals for cost, QALYs and net benefits calculated at $£ 20,000$ per QALY. We also report incremental net benefit relative to strategy 1 (selective scanning and induction of labour for SGA and LGA). Decision uncertainty is illustrated with cost-effectiveness acceptability curves (CEACs). All costs were from a third-party (payer) perspective (the English NHS), and the health consequences from a foetal perspective only. All costs and QALYs were discounted by $3.5 \%$ annually, as recommended by NICE. ${ }^{64}$ The time-horizon was 20 years in the base-case scenario. Costs in other currencies were converted to GBP ( $f$ ) by the exchange rate of the respective year. All prices were updated to the price level of 2016/17 using the hospital \& community health services $(\mathrm{HCHS})$ index..$^{56}$

To complement the probabilistic sensitivity analysis, we also investigated the model's sensitivity to key parameters through one-way sensitivity analysis. Further, our base case analysis assumed early labour induction would only affect long-term foetal outcomes via its impact upon neonatal outcomes. However, there is evidence suggesting that induction of labour may of itself increase the risk of special educational needs in later life. ${ }^{40}$ We therefore explore the impact of an independent effect of induction of labour on the risk of special educational needs.

We report the per-patient (i.e. per mother/infant dyad) and population Expected Value of Perfect Information (EVPI) at a willingness to pay of $f 20,000 / \mathrm{QALY}$ and the Expected Value of Perfect Parameter Information (EVPPI) for each parameter individually using the Sheffield Accelerated Value 
of Information (SAVI) tool. ${ }^{65}$ Parameters with a positive EVPPI were grouped into those which could be collected within a single research study and the EVPPI for that group of parameters calculated. The Expected Value of Sample Information (EVSI) for any parameters or groups of parameters was then calculated using the method of Moment Matching with 30 nested samples. ${ }^{66}$ EVPPI and EVSI calculations are traditionally extremely computationally expensive. The SAVI and moment matching methods generate statistical approximations, allowing calculation within a feasible timeframe. Briefly, SAVI estimates the EVPPI via a generalised additive model (GAM) with non-parametric smoothing applied to the sampled input parameter set and resulting net benefits. Our implementation of the $\mathrm{MM}$ method relies on the conjugate distribution of the respective prior to estimate the preposterior distribution for a given study sample size (see Appendix 3 for code and walk-through). Population values are calculated over a time horizon of 10 years and as a 'conservative' estimate, assuming the information is only of value to singleton nulliparous pregnancies resulting in a beneficial population of $1,689,663$ and again with a broader estimate which assumes the information is of value to all pregnancies in England ( $n=5,477,940$, Appendix 2).

The model was coded in $\mathrm{R}^{67}$ and associated packages. ${ }^{68-74}$ Full model code is available from the corresponding author upon request. 


\section{Results}

Economic evaluation results are presented based on 100,000 simulations of the model. Value of information analysis statistics are based on 10,000 simulations (stability testing results reported in Appendix 4).

Given current evidence and assuming a willingness to pay of $£ 20,000$ per QALY, the strategy associated with the highest net benefit is strategy 3: a presentation-only scan for all women (unless further screening is clinically indicated) with induction of labour where LGA or SGA are suspected. The added benefits from universal ultrasound screening for foetal size are unlikely to justify its added cost (Table 2). However, there is substantial uncertainty associated with this recommendation, with only a $44 \%$ probability of this yielding the highest net benefit, and a 39\% probability of universal screening being optimal (Table 2, Figure 2). As the willingness to pay threshold rises, the probability that universal screening becomes the most cost-effective strategy also rises (Figure 2).

One-way sensitivity analyses suggested that the cost-effectiveness outcomes were only sensitive to a few parameters: presentation only scanning is the most cost-effective option if the time horizon of the analysis is below 45 years, above which universal screening becomes the most cost-effective option. A presentation-only scan remains the most cost-effective option provided it costs no more than 190 , above which status quo is the most cost-effective, and that the baseline stillbirth rate is above $0.28 \%$, at which point universal scanning is most cost-effective. Finally, we found that the impact of induction of labour on risk of special educational needs (SEN) would only change the conclusions if the relative risk of SEN was lower than 0.95 , or above 1.3; observational data suggest that the effect is highly unlikely to be outside this range (Appendix 5). ${ }^{40}$

The per patient expected value of perfect information (EVPI) is $£ 31.56$. Given a population who can benefit from the information of 1,689,663 (see Appendix 2), the population EVPI to England is $f 53.3 \mathrm{~m}$. If the results of the analysis are assumed generalizable to all pregnancies in England, then the population EVPI is $f 172.9 \mathrm{~m}$ (Table 3). Only five input parameters yielded a population EVPPI greater 
than $f 100,000$, and these logically group into three clusters of outcome measures that could be collected in possible future studies or RCTs (labelled studies 1, 2 and 3). The parameter with the greatest EVPPI is the difference in net cost of induced versus non-induced deliveries, accounting for $84 \%$ of the EVPI. No other parameters individually account for more than $1 \%$ of the total EVPI (Table $3)$.

EVSI analysis of study 1 , exploring the net cost difference between early labour induction and expectant management suggests scope for it to yield a positive return on investment. For example, a study with 1000 patients (in each arm of a two-arm study) has an EVSI to England of $£ 11.3 \mathrm{~m}$ (or $£ 97.2 \mathrm{~m}$ if this information is of value to all pregnancies in England, not just low risk nulliparous singleton pregnancies, Figure 3 ). If such a study were to cost $f 1 \mathrm{~m}$, it would yield a net return on investment (ENGS) of at least $f 10.3 \mathrm{~m}$. The EVSI algorithm was not able to estimate an EVSI for studies 2 and 3; following investigation, we concluded that for very low EVSIs, the approximation method is not able to return a value. We therefore conclude that the EVSI is very low and thus studies collecting data on the respective parameters are unlikely to be worth more than the cost of collecting them. 


\section{Discussion}

Given current information, the most cost-effective strategy for late-pregnancy ultrasound scanning is to offer all women a presentation-only scan (those women who are currently indicated to undergo a full third-trimester ultrasound scan to continue to do so), and where SGA or LGA are suspected, the mother should be offered induction of labour, unless otherwise contraindicated. Given current thresholds, ${ }^{64}$ universal routine ultrasound screening to assess foetal size is not cost-effective.

There is substantial decision uncertainty around this recommendation. However, the expected value of eliminating all uncertainty is only worth a maximum of $f 172.9 \mathrm{~m}$, or 8644 QALYs to the population of England (assuming $£ 20,000$ per QALY). This represents the expected opportunity loss due to the probability that the above recommendation is incorrect (crudely, the probability of being 'wrong' multiplied by the consequence of being 'wrong'). The majority of the EVPI is concentrated in a single parameter, namely the difference in cost as a result of early induction of labour. This is somewhat surprising, but arises due to the large standard error around the relevant model parameter (Appendix 2, Table A2.1, row "Induction of labour"). This is because the cost encompasses not only the cost of inducing a pregnancy itself, but the costs of delivery and antenatal visits which may or may not be avoided too. Induction also has an uncertain impact on complications and hence long-term cost and outcomes of delivery. On top of this, less than perfect sensitivity and specificity of the scans at detecting LGA and SGA babies magnify the impact of uncertainty in the cost and outcomes of induction of labour. The EVSI of this parameter suggests that a study of 'reasonable' size (eg 1000 mothers per arm with a cost of $f 1 \mathrm{~m}$ ) would likely yield a highly positive return on investment.

An ideal study design to measure the cost-difference would be a study randomising mothers to induction of labour or not, irrespective of indication. This is likely to raise ethical issues and would require careful consideration of the pros and cons and risks to mothers and their babies, based on current state of knowledge. A non-randomised study design (eg database or cohort analysis) would be feasible but at risk of bias. The mathematics of value of information analysis are blind to whether 
reducing uncertainty in a parameter is ethical or not, or even possible or not. Instead, as with all economic evaluation, they provide a guide and input to the decision-making process. An important finding from our analysis is that there is no evidence that a large scale RCT powered to detect a difference in stillbirth would be a worthwhile investment: the EVPPI from reducing uncertainty in stillbirth rates is worth less than $£ 100,000$, a sum for which it is not possible to deliver an RCT.

We believe our analysis represents the most plausible summary of the evidence on the costs and effects of different ultrasound screening and subsequent management strategies in late pregnancy. The decision model translates uncertainty in parameters (crudely, the standard errors around mean estimates of effect, cost and health state utilities) to decision uncertainty (standard errors around mean estimates of net benefit). The value of information analysis then predicts the likely return on investment from reducing the SEs of the input parameters.

However, the validity of our conclusion rests entirely on the validity of the model. Whilst we believe we have appropriately captured parameter uncertainty, we have implicitly assumed that the structure of the model itself is 'correct'. Addressing such structural uncertainty is challenging in decision models. In theory it would require constructing many alternative models and comparing or averaging out the results, which would be prohibitively expensive. However, where possible we did explore structural uncertainty, for example our base case assumed that all long-term morbidity was mediated through the risk of neonatal morbidity, whilst there is evidence to suggest an independent effect of induction of labour on risk of special educational needs. We explored this and found our conclusions to be robust to all but implausibly extreme assumptions as to the relative risk. Our analytic perspective was limited to foetal outcomes only, excluding maternal quality of life. This may underestimate the QALY gains from screening and so underestimate cost-effectiveness.

Secondly, our conclusions regarding the cost-effectiveness of presentation scanning are contingent on midwifes being able to undertake the scan as part of a routine antenatal contact. This is currently unknown and requires a feasibility study to test. It should also be noted that the scans will certainly 
increase the burden on midwifes whilst we predict a reduction in delivery complications. This will require a shift in resources from secondary care to (antenatal) midwifery. The budgetary mechanisms underlying this are not considered in our analysis. It is worth noting that our previous work ${ }^{14}$ focusing only on presentation scans (and not including the alternative strategies considered here) concluded that a presentation-only scan was cost-effective so long as it could be provided for $£ 19.80$ or less. Our analysis here, which models longer term costs and outcomes in greater detail, suggests greater scope for cost-effectiveness, with our one-way sensitivity analysis suggesting the scan remaining costeffective so long as it can be provided for less than approximately $f 90$ (Appendix 5, Figure A4.2).

To our knowledge this is the first value of information analysis estimating the return on investment from future research into late pregnancy ultrasound scans. Economic evaluations of obstetric investigations commonly include estimates of the value of perfect information, for example there may be value in future studies on quality of life gains and costs of early detection of gestational diabetes, ${ }^{75}$ the effects of interventions to prevent postnatal depression, ${ }^{76}$ the cost-effectiveness of financial incentives for smoking cessation during pregnancy, ${ }^{77}$ and possibly into the effectiveness of a screening programme to reduce periconceptional exposure to methylmercury. ${ }^{78}$ However, we are not aware of any attempts to calculate the expected value of sample information from specific study designs in obstetrics.

\section{Conclusion}

Our results suggest that universal ultrasound for foetal presentation only may be both clinically and economically justified, but that implementation research is needed before it is adopted into routine care. Specifically, this must explore whether a scan can be conducted by a midwife during a routine antenatal visit. Universal ultrasound including estimation of foetal weight is of borderline costeffectiveness, and sensitive to certain assumptions. Our formal value of information analysis suggests that future research should be focused on the net cost of induction of labour compared to expectant 
management, and that there is unlikely to be value in a large scale RCT of routine vs selective ultrasound screening powered to detect a difference in stillbirth rates. 


\section{References}

1. GBD 2016 Causes of Death Collaborators. Global, regional, and national age-sex specific mortality for 264 causes of death, 1980-2016: a systematic analysis for the Global Burden of Disease Study 2016. Lancet (London, England) 2017;390(10100):1151-210. Dol: 10.1016/s01406736(17)32152-9

2. Office for National Statistics. Births in England and Wales: summary tables, release 31st July 2019 2019 [Available from: https://www.ons.gov.uk/peoplepopulationandcommunity/birthsdeathsandmarriages/livebir ths/datasets/birthsummarytables.

3. Gardosi J, Madurasinghe V, Williams M, et al. Maternal and fetal risk factors for stillbirth: population based study. BMJ (Clinical research ed) 2013;346:f108-f08. Dol: 10.1136/bmj.f108

4. National Institute for Health and Care Excellence. Clinical guideline: Antenatal care for uncomplicated pregnancies. In: National Institute for Health and Care Excellence, ed., 2008.

5. Royal College of Obstetrics \& Gynaecologists. The Investigation and Management of the Small-forGestational-Age Fetus (Green-top guideline No. 31). In: Royal College of Obstetrics \& Gynaecologists, ed., 2013.

6. Henrichs J, Verfaille V, Viester L, et al. Effectiveness and cost-effectiveness of routine third trimester ultrasound screening for intrauterine growth restriction: study protocol of a nationwide stepped wedge cluster-randomized trial in The Netherlands (The IRIS Study). BMC Pregnancy Childbirth 2016;16(1):310. Dol: 10.1186/s12884-016-1104-8

7. Le Ray $\mathrm{C}$, Lacerte $\mathrm{M}$, Iglesias $\mathrm{MH}$, et al. Routine third trimester ultrasound: what is the evidence? J Obstet Gynaecol Can 2008;30(2):118-22

8. Le Ray C, Morin L. Routine versus indicated third trimester ultrasound: is a randomized trial feasible? J Obstet Gynaecol Can 2009;31(2):113-9

9. Bricker L, Medley N, Pratt JJ. Routine ultrasound in late pregnancy (after 24 weeks' gestation). The Cochrane database of systematic reviews 2015(6):CD001451. Dol: 10.1002/14651858.CD001451.pub4

10. Smith GCS. Universal screening for foetal growth restriction. Best practice \& research Clinical obstetrics \& gynaecology 2018;49:16-28. Dol: 10.1016/j.bpobgyn.2018.02.008

11. Pasupathy D, Dacey A, Cook E, et al. Study protocol. A prospective cohort study of unselected primiparous women: the pregnancy outcome prediction study. BMC Pregnancy Childbirth 2008;8:51. Dol: 10.1186/1471-2393-8-51

12. Sovio $U$, White IR, Dacey A, et al. Screening for fetal growth restriction with universal third trimester ultrasonography in nulliparous women in the Pregnancy Outcome Prediction (POP) study: a prospective cohort study. Lancet (London, England) 2015;386(10008):2089-97. Dol: 10.1016/s0140-6736(15)00131-2

13. Sovio U, Moraitis AA, Wong HS, et al. Universal vs selective ultrasonography to screen for largefor-gestational-age infants and associated morbidity. Ultrasound in obstetrics \& gynecology : the official journal of the International Society of Ultrasound in Obstetrics and Gynecology 2018;51(6):783-91. Dol: 10.1002/uog.17491

14. Wastlund D, Moraitis AA, Dacey A, et al. Screening for breech presentation using universal latepregnancy ultrasonography: A prospective cohort study and cost effectiveness analysis. PLoS Med 2019;16(4):e1002778. Dol: 10.1371/journal.pmed.1002778

15. Wastlund D, Moraitis AA, Thornton JG, et al. The cost-effectiveness of universal late-pregnancy screening for macrosomia in nulliparous women: a decision analysis. BJOG : an international journal of obstetrics and gynaecology 2019;126(10):1243-50. Dol: 10.1111/1471-0528.15809

16. Wastlund D, Wilson ECF, Moraitis AA, et al. A cost-effectiveness analysis of universal late pregnancy ultrasound in nulliparous women to detect small-for-gestational-age fetuses. [Manuscript in preparation]

17. Pratt J, Raiffa H, Schlaifer R. Introduction to Statistical Decision Theory. Cambridge, MA: Massachusetts Institute of Technology, 1995. 
18. Claxton K. The irrelevance of inference: a decision-making approach to the stochastic evaluation of health care technologies. Journal of health economics 1999;18(3):341-64. Dol: S01676296(98)00039-3 [pii]

19. Wilson EC. A practical guide to value of information analysis. Pharmacoeconomics 2015;33(2):10521. Dol: $10.1007 / \mathrm{s} 40273-014-0219-x$

20. Heazell AE, Hayes DJ, Whitworth M, et al. Biochemical tests of placental function versus ultrasound assessment of fetal size for stillbirth and small-for-gestational-age infants. The Cochrane database of systematic reviews 2019;5:Cd012245. Dol: 10.1002/14651858.CD012245.pub2

21. Monier I, Blondel B, Ego A, et al. Poor effectiveness of antenatal detection of fetal growth restriction and consequences for obstetric management and neonatal outcomes: a French national study. BJOG : an international journal of obstetrics and gynaecology 2015;122(4):51827. Dol: $10.1111 / 1471-0528.13148$

22. Grobman WA, Rice MM, Reddy UM, et al. Labor Induction versus Expectant Management in LowRisk Nulliparous Women. N Engl J Med 2018;379(6):513-23. Dol: 10.1056/NEJMoa1800566

23. Blackwell SC, Refuerzo J, Chadha R, et al. Overestimation of fetal weight by ultrasound: does it influence the likelihood of cesarean delivery for labor arrest? American journal of obstetrics and gynecology 2009;200(3):340.e1-3. Dol: 10.1016/j.ajog.2008.12.043

24. Middleton P, Shepherd E, Crowther CA. Induction of labour for improving birth outcomes for women at or beyond term. The Cochrane database of systematic reviews 2018;5:Cd004945. Dol: 10.1002/14651858.CD004945.pub4

25. Leung WC, Pun TC, Wong WM. Undiagnosed breech revisited. British journal of obstetrics and gynaecology 1999;106(7):638-41

26. Ben-Meir A, Elram T, Tsafrir A, et al. The incidence of spontaneous version after failed external cephalic version. American journal of obstetrics and gynecology 2007;196(2):157.e1-3. Dol: 10.1016/j.ajog.2006.10.889

27. Morrison JJ, Rennie JM, Milton PJ. Neonatal respiratory morbidity and mode of delivery at term: influence of timing of elective caesarean section. British journal of obstetrics and gynaecology 1995;102(2):101-6

28. Ouzounian JG, Gherman RB. Shoulder dystocia: are historic risk factors reliable predictors? American journal of obstetrics and gynecology 2005;192(6):1933-5; discussion 35-8. Dol: 10.1016/j.ajog.2005.02.054

29. Moraitis AA, Wood AM, Fleming $M$, et al. Birth weight percentile and the risk of term perinatal death. Obstetrics and gynecology 2014;124(2 Pt 1):274-83. Dol: 10.1097/aog.0000000000000388

30. Rossi AC, Mullin P, Prefumo F. Prevention, management, and outcomes of macrosomia: a systematic review of literature and meta-analysis. Obstetrical \& gynecological survey 2013;68(10):702-9. Dol: 10.1097/01.ogx.0000435370.74455.a8

31. Chongsuvivatwong V, Bachtiar $\mathrm{H}$, Chowdhury ME, et al. Maternal and fetal mortality and complications associated with cesarean section deliveries in teaching hospitals in Asia. The journal of obstetrics and gynaecology research 2010;36(1):45-51. Dol: 10.1111/j.14470756.2009.01100.x

32. Gibson KS, Waters TP, Bailit JL. Maternal and neonatal outcomes in electively induced low-risk term pregnancies. American journal of obstetrics and gynecology 2014;211(3):249.e1-49.e16. Dol: 10.1016/j.ajog.2014.03.016

33. Boulvain M, Senat MV, Perrotin F, et al. Induction of labour versus expectant management for large-for-date fetuses: a randomised controlled trial. Lancet (London, England) 2015;385(9987):2600-5. Dol: 10.1016/s0140-6736(14)61904-8

34. MacKenzie IZ, Shah M, Lean K, et al. Management of shoulder dystocia: trends in incidence and maternal and neonatal morbidity. Obstetrics and gynecology 2007;110(5):1059-68. Dol: 10.1097/01.AOG.0000287615.35425.5c 
35. Sandmire HF, DeMott RK. The Green Bay cesarean section study. IV. The physician factor as a determinant of cesarean birth rates for the large fetus. American journal of obstetrics and gynecology 1996;174(5):1557-64. Dol: 10.1016/s0002-9378(96)70606-3

36. Stock SJ, Ferguson E, Duffy A, et al. Outcomes of elective induction of labour compared with expectant management: population based study. BMJ (Clinical research ed) 2012;344:e2838. Dol: $10.1136 / \mathrm{bmj} . e 2838$

37. Thorngren-Jerneck K, Herbst A. Low 5-minute Apgar score: a population-based register study of 1 million term births. Obstetrics and gynecology 2001;98(1):65-70

38. Hofmeyr GJ, Hannah M, Lawrie TA. Planned caesarean section for term breech delivery. The Cochrane database of systematic reviews 2015(7):CD000166. Dol: 10.1002/14651858.CD000166.pub2

39. Pasupathy D, Wood AM, Pell JP, et al. Time trend in the risk of delivery-related perinatal and neonatal death associated with breech presentation at term. Int J Epidemiol 2009;38(2):4908. Dol: $10.1093 /$ ije/dyn225

40. MacKay DF, Smith GC, Dobbie R, et al. Gestational age at delivery and special educational need: retrospective cohort study of 407,503 schoolchildren. PLoS Med 2010;7(6):e1000289. Dol: 10.1371/journal.pmed.1000289

41. Persson M, Razaz N, Tedroff K, et al. Five and 10 minute Apgar scores and risks of cerebral palsy and epilepsy: population based cohort study in Sweden. BMJ (Clinical research ed) 2018;360:k207. Dol: 10.1136/bmj.k207

42. Iliodromiti S, Mackay DF, Smith GC, et al. Apgar score and the risk of cause-specific infant mortality: a population-based cohort study. Lancet (London, England) 2014;384(9956):1749-55. Dol: 10.1016/s0140-6736(14)61135-1

43. Department of Health and Social Care. NHS reference costs 2016 to 20172016 [Available from: https://improvement.nhs.uk/resources/reference-costs/\#rc1718.

44. Vijgen SM, Boers KE, Opmeer BC, et al. Economic analysis comparing induction of labour and expectant management for intrauterine growth restriction at term (DIGITAT trial). Eur J Obstet Gynecol Reprod Biol 2013;170(2):358-63. Dol: 10.1016/j.ejogrb.2013.07.017

45. Palencia R, Gafni A, Hannah ME, et al. The costs of planned cesarean versus planned vaginal birth in the Term Breech Trial. CMAJ : Canadian Medical Association journal = journal de I'Association medicale canadienne 2006;174(8):1109-13. Dol: 10.1503/cmaj.050796

46. James $M$, Hunt $K$, Burr $R$, et al. A decision analytical cost analysis of offering ECV in a UK district general hospital. BMC Health Serv Res 2001;1:6. Dol: 10.1186/1472-6963-1-6

47. Alfirevic Z, Keeney E, Dowswell T, et al. Which method is best for the induction of labour? A systematic review, network meta-analysis and cost-effectiveness analysis. Health Technol Assess 2016;20(65):1-584. Dol: 10.3310/hta20650

48. Culligan PJ, Myers JA, Goldberg RP, et al. Elective cesarean section to prevent anal incontinence and brachial plexus injuries associated with macrosomia--a decision analysis. International urogynecology journal and pelvic floor dysfunction 2005;16(1):19-28; discussion 28. Dol: 10.1007/s00192-004-1203-3

49. Mistry $H$, Heazell $A E$, Vincent $O$, et al. A structured review and exploration of the healthcare costs associated with stillbirth and a subsequent pregnancy in England and Wales. BMC Pregnancy Childbirth 2013;13:236. Dol: 10.1186/1471-2393-13-236

50. Barrett B, Mosweu I, Jones CR, et al. Comparing service use and costs among adolescents with autism spectrum disorders, special needs and typical development. Autism 2015;19(5):562-9. Dol: $10.1177 / 1362361314536626$

51. Access Economics. The Economic Impact of Cerebral Palsy in Australia in 2007. Sydney, NSW, 2008.

52. Young NL, Rochon TG, McCormick A, et al. The health and quality of life outcomes among youth and young adults with cerebral palsy. Arch Phys Med Rehabil 2010;91(1):143-8. Dol: 10.1016/j.apmr.2009.08.152 
53. Szende A, Janssen B, Cabasés J. Self-Reported Population Health: An International Perspective based on EQ-5D: Springer Dordrecht Heidelberg New York London, 2014.

54. Leigh S, Granby $P$, Turner $M$, et al. The incidence and implications of cerebral palsy following potentially avoidable obstetric complications: a preliminary burden of disease study. BJOG : an international journal of obstetrics and gynaecology 2014;121(13):1720-8. Dol: 10.1111/1471-0528.12897

55. NHS Digital. NHS Staff Earnings - Estimates ot September 2017, Provisional Statistics, 2017.

56. Curtis L, Burns A. Unit Costs of Health and Social Care 2017: Personal Social Services Research Unit, 2017.

57. Curtis L. Unit Costs of health and Social Care 2008. Cornwallis Building, The University of Kent, Canterbury, Kent CT2 7NF: Personal Social Services Research Unit, 2008.

58. NHS Purchasing and Supply Agency. Cost-effectiveness of ultrasound elastography in the assessment of liver fibrosis, 2009.

59. Malloy MH, Freeman DH. Respiratory distress syndrome mortality in the United States, 1987 to 1995. J Perinatol 2000;20(7):414-20

60. Curtis L, Burns A. Unit costs of health and social care 2016: Personal Social Services Research Unit, 2016.

61. Office for National Statistics. National Life Tables, United Kingdom, 1980-82 to 2014-16: Office for National Statistics, 2017.

62. Palisano R, Rosenbaum $P$, Walter $S$, et al. Development and reliability of a system to classify gross motor function in children with cerebral palsy. Dev Med Child Neurol 1997;39(4):214-23

63. Health and Social Care Information Centre. NHS Maternity Statistics 2016-17: NHS Digital, 2017.

64. National Institute for Health and Care Excellence. Guide to the methods of technology appraisal 2013 London: NICE; 2013 [Available from: http://www.nice.org.uk/media/D45/1E/GuideToMethodsTechnologyAppraisal2013.pdf.

65. Strong M, Oakley JE, Brennan A. Estimating multiparameter partial expected value of perfect information from a probabilistic sensitivity analysis sample: a nonparametric regression approach. Medical decision making : an international journal of the Society for Medical Decision Making 2014;34(3):311-26. Dol: 10.1177/0272989X13505910

66. Heath A, Baio G. Calculating the Expected Value of Sample Information Using Efficient Nested Monte Carlo: A Tutorial. Value Health 2018;21(11):1299-304. Dol: 10.1016/j.jval.2018.05.004

67. R Core Team. R: A Language and Environment for Statistical Computing. Vienna, Austria: R Foundation for Statistical Computing, 2017.

68. Baio G, Berardi A, Heath A. BCEA: Bayesian Cost Effectiveness Analysis, 2018.

69. Fan FY. FinCal: Time Value of Money, Time Series Analysis and Computational Finance. R package version 063, 2016.

70. Wickham H. ggplot2: Elegant Graphics for Data Analysis: Springer-Verlag New York, 2016.

71. Warnes G, Bolker B, Lumley T. gtools: Various R Programming Tools, 2018.

72. Wickham H, Bryan J. readxl: Read Excel Files, 2018.

73. Wickham H, Henry L. tidyr: Easily Tidy Data with 'spread()' and 'gather()' Functions, 2019.

74. Strong M. SAVI: SAVI Sheffield Accelerated Value of Information, 2015.

75. Farrar D, Simmonds $M$, Griffin S, et al. The identification and treatment of women with hyperglycaemia in pregnancy: an analysis of individual participant data, systematic reviews, meta-analyses and an economic evaluation. Health Technol Assess 2016;20(86):1-348. Dol: 10.3310/hta20860

76. Morrell CJ, Sutcliffe $P$, Booth A, et al. A systematic review, evidence synthesis and meta-analysis of quantitative and qualitative studies evaluating the clinical effectiveness, the costeffectiveness, safety and acceptability of interventions to prevent postnatal depression. Health Technol Assess 2016;20(37):1-414. Dol: 10.3310/hta20370

77. Boyd KA, Briggs AH, Bauld L, et al. Are financial incentives cost-effective to support smoking cessation during pregnancy? Addiction 2016;111(2):360-70. Dol: 10.1111/add.13160 
78. Gaskin J, Rennie C, Coyle D. Reducing Periconceptional Methylmercury Exposure: Cost-Utility Analysis for a Proposed Screening Program for Women Planning a Pregnancy in Ontario, Canada. Environmental health perspectives 2015;123(12):1337-44. Dol: 10.1289/ehp.1409034 
Tables and Figures

Figure 1. Model structure overview: Screening-management options and foetal conditions.

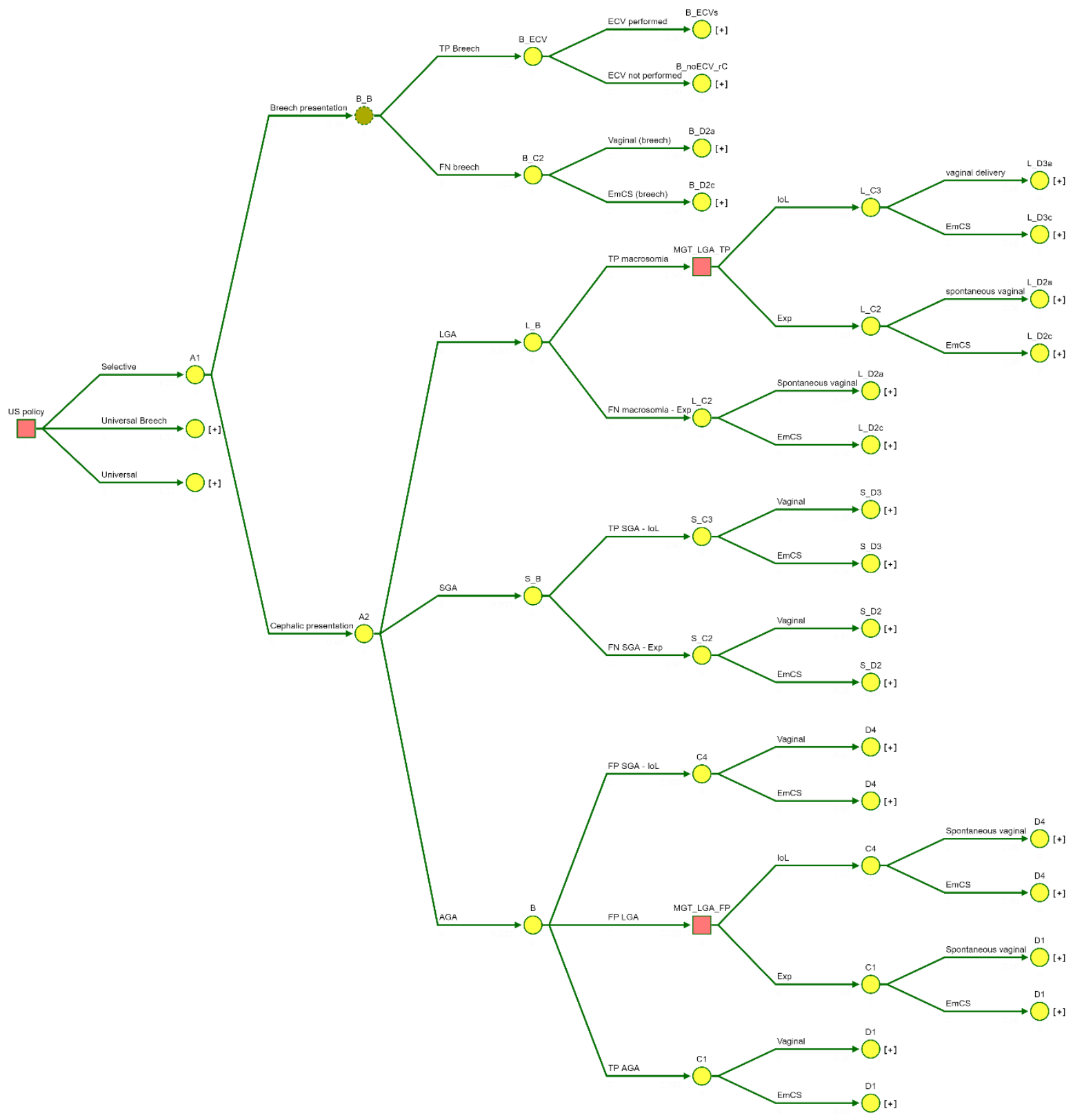


[+] = sub-branches of model collapsed for clarity; see Appendix 1 for expanded nodes. Nodes with the same letter have identical subsequent structures, whilst a different number and lowercase letter indicates different probabilities assigned to the next subbranch. The prefix before the underscore indicates a set of probabilities relevant to breech (B_), LGA (L_) or SGA (S_) For example, nodes D1 and D4 have identical sub-structures, but D1 relates to AGA babies delivered spontaneously, whereas D4 relates to AGA babies wrongly diagnosed as SGA or LGA and undergoing induction of labour unnecessarily. US = ultrasound; TP = true positive; $F N=$ false negative; $F P=$ false positive; $T N$ = true negative; $\mathrm{ECV}$ = external cephalic version; EmCS = Emergency Caesarean section; $\mathrm{Exp}=$ Expectant management; loL = Induction of labour 
Figure 2. Cost-effectiveness acceptability curves showing probability of cost-effectiveness as a function of willingness-to-pay for an additional quality-adjusted life year.

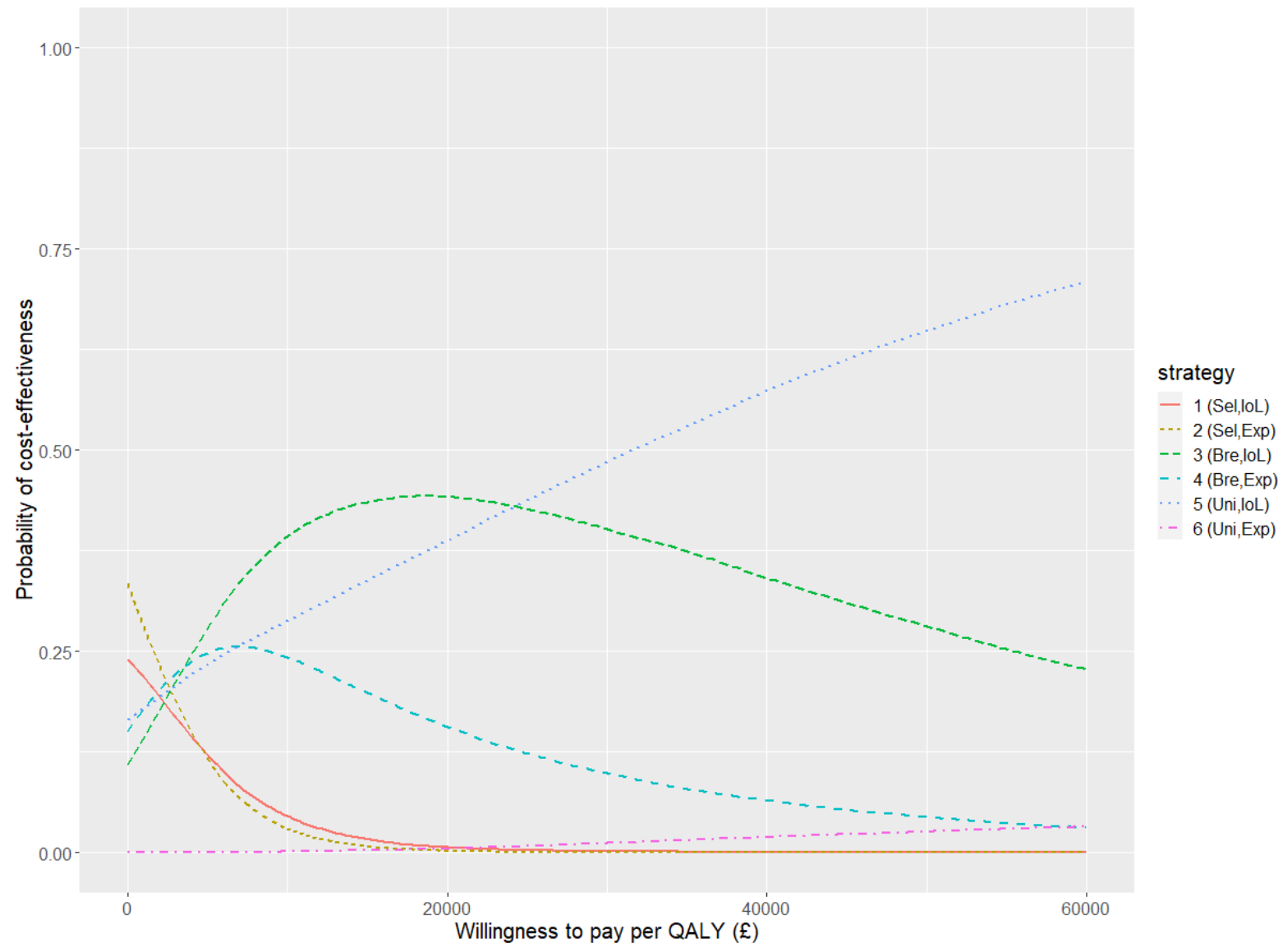

Sel = selective scanning; Bre = Universal presentation-only scan; Uni = Universal scan of foetal biometry and presentation; $I O L=$ Induction of labour if $L G A$ suspected; Exp = Expectant management if $L G A$ suspected. 
Figure 3: Expected Value of Sample Information of Study 1.

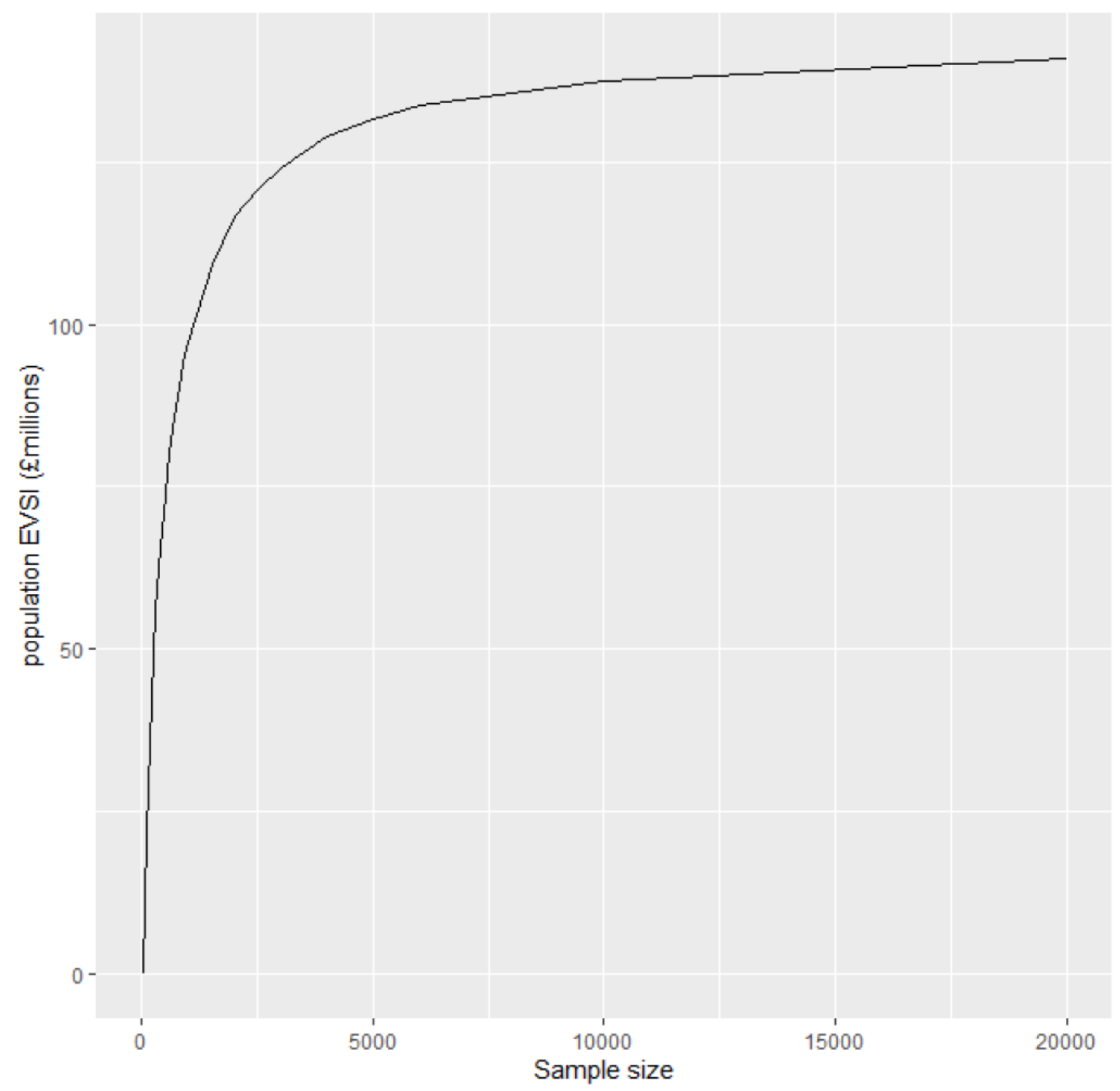

Expected value of sample information as a function of sample size for a study of the cost-difference between early induction of labour versus expectant management. 
Table 1: Comparator strategies / policies

\begin{tabular}{lllll} 
Strategy & Screen & \multicolumn{3}{c}{ Offered management if diagnosed: } \\
& & Breech+ & Macrosomia+ & SGA+ \\
\hline 1 & Selective & ECV & loL & loL \\
2 & Selective & ECV & Exp & loL \\
3 & Universal Breech & ECV & loL & loL \\
4 & Universal Breech & ECV & Exp & loL \\
5 & Universal & ECV & loL & loL \\
6 & Universal & ECV & Exp & loL
\end{tabular}

$E C V=$ External cephalic version; Exp $=$ Expectant management $; \mathrm{loL}=$ Induction of labour; $S G A=$ Small for gestational age 
Table 2. Cost effectiveness results (per mother scanned).

\begin{tabular}{|c|c|c|c|c|c|c|}
\hline \multicolumn{2}{|c|}{ Screening + management } & Cost $(f)$ & QALYS & $N B \mid f 20 k$ & INB $\mid £ 20 k$ & P_CE $\mid f 20 k$ \\
\hline 1. & Selective US + IoL for LGA & $\begin{array}{l}6090 \\
(4420,7890)\end{array}$ & $\begin{array}{l}13.640 \\
(13.441,13.841)\end{array}$ & $\begin{array}{l}f 266,719 \\
(£ 262,333, £ 271,079)\end{array}$ & $\begin{array}{l}f 0 \\
(£ 0, f 0)\end{array}$ & $0.65 \%$ \\
\hline 2. & Selective US + Exp for LGA & $\begin{array}{l}6091 \\
(4424,7889)\end{array}$ & $\begin{array}{l}13.639 \\
(13.439,13.839)\end{array}$ & $\begin{array}{l}£ 266,682 \\
(£ 262,297, £ 271,040)\end{array}$ & $\begin{array}{l}-£ 37.09 \\
(-£ 124.7, £ 35.24)\end{array}$ & $0.22 \%$ \\
\hline 3. & $\begin{array}{l}\text { Universal US for breech + } \\
+ \text { loL for LGA * }\end{array}$ & $\begin{array}{l}6101 \\
(4443,7887)\end{array}$ & $\begin{array}{l}13.645 \\
(13.446,13.846)\end{array}$ & $\begin{array}{l}£ 266,806 \\
(£ 262,426, f 271,154)\end{array}$ & $\begin{array}{l}£ 87.36 \\
(£ 4.88, £ 205.68)\end{array}$ & $44.19 \%$ \\
\hline 4. & $\begin{array}{l}\text { Universal US for breech + } \\
\text { Exp for LGA }\end{array}$ & $\begin{array}{l}6102 \\
(4446,7887)\end{array}$ & $\begin{array}{l}13.644 \\
(13.444,13.844)\end{array}$ & $\begin{array}{l}£ 266,769 \\
(£ 262,389, £ 271,120)\end{array}$ & $\begin{array}{l}f 50.29 \\
(-f 68.06, f 186.43)\end{array}$ & $15.63 \%$ \\
\hline 6. & Universal US + Exp for LGA & $\begin{array}{l}6178 \\
(4508,7972)\end{array}$ & $\begin{array}{l}13.646 \\
(13.446,13.846)\end{array}$ & $\begin{array}{l}£ 266,734 \\
(£ 262,351, £ 271,099)\end{array}$ & $\begin{array}{l}£ 14.47 \\
(-£ 133.98, f 173.31)\end{array}$ & $0.51 \%$ \\
\hline 5. & Universal US + IoL for LGA & $\begin{array}{l}6180 \\
(4498,7983)\end{array}$ & $\begin{array}{l}13.648 \\
(13.448,13.849)\end{array}$ & $\begin{array}{l}f 266,779 \\
(£ 262,386, £ 271,147)\end{array}$ & $\begin{array}{l}£ 60.24 \\
(-f 151.43, f 281.7)\end{array}$ & $38.81 \%$ \\
\hline
\end{tabular}

Strategies are listed in order of increasing cost $(1,2,3,4,6,5)$. Management refers to management strategy when LGA is suspected, all cases of suspected SGA are assumed induced and breech to be offered ECV. * Strategy with highest expected net benefit (shown in bold). IoL = Induction of Labour; Exp = Expectant Management; INB = Incremental net benefit relative to current practice (strategy 1 , selective US + induction of labour); $N B=$ Net benefit $; P_{-} C E / E 20 \mathrm{k}=$ Probability of being the most cost effective strategy given a willingness to pay of $£ 20,000$ per QALY gained. 
Table 3. Expected value of perfect information.

\begin{tabular}{|c|c|c|c|c|}
\hline & $\begin{array}{l}\text { Per Person EVPPI } \\
\text { (fexpected, SE) }\end{array}$ & $\begin{array}{l}\% \text { of } \\
\text { EVPI }\end{array}$ & pEVPPI $(f)^{*}$ & pEVPPI $(f)^{*}$ \\
\hline \multicolumn{5}{|l|}{ Study 1} \\
\hline Cost difference from early induction of labour & $26.51(0.07)$ & 84 & $44,790,000$ & $145,200,000$ \\
\hline \multicolumn{5}{|l|}{ Study 2} \\
\hline$R R$ for acidosis in macrosomic fetuses if induced early & $0.27(0.04)$ & $1 \%$ & 456,000 & $1,478,000$ \\
\hline OR for mortality if fetus is macrosomic & $0.26(0.03)$ & $1 \%$ & 438,900 & $1,423,000$ \\
\hline Group & $0.72(0.07)$ & $2 \%$ & $1,215,199$ & $3,939,513$ \\
\hline \multicolumn{5}{|l|}{ Study 3} \\
\hline $\begin{array}{l}\text { RR for emergency CS among SGA fetuses following } \\
\text { early labour induction }\end{array}$ & $0.06(0.01)$ & $0 \%$ & 99,290 & 321,900 \\
\hline \multirow{2}{*}{$\begin{array}{l}\text { OR for severe neonatal morbidity if fetus is SGA } \\
\text { Group }\end{array}$} & $0.03(0.01)$ & $0 \%$ & 48,740 & 158,000 \\
\hline & $0.26(0.04)$ & $1 \%$ & 443,104 & $1,436,484$ \\
\hline Expected Value of Perfect Information & $31.56(-)$ & $100 \%$ & $53,326,764$ & $172,883,786$ \\
\hline \multicolumn{5}{|c|}{ * First pEVPPI column assumes information is applicable just to the target population (nulliparous } \\
\hline \multicolumn{5}{|c|}{ singleton pregnancies), second assumes the information is equally applicable to all births in England. } \\
\hline \multicolumn{5}{|c|}{$C S=$ Caesarean section; EVPI = Expected value of information; EVPPI = Expected value of partial perfect } \\
\hline \multicolumn{5}{|c|}{ information; $O R=$ Odds ratio; $S E=$ Standard error; $R R=$ Relative risk. Standard error around estimates } \\
\hline \multicolumn{5}{|c|}{ of EVPPI are a result of the SAVI ${ }^{74}$ approximation algorithm. The EVPI is calculated directly and thus } \\
\hline \multicolumn{5}{|c|}{ has no associated standard error. Note sum of EVPPI will not usually equal the EVPI due to interactions } \\
\hline
\end{tabular}




\section{Appendix 1: Model structure - Breech, LGA and SGA}

Figure A1.1. Outcomes associated with breech.

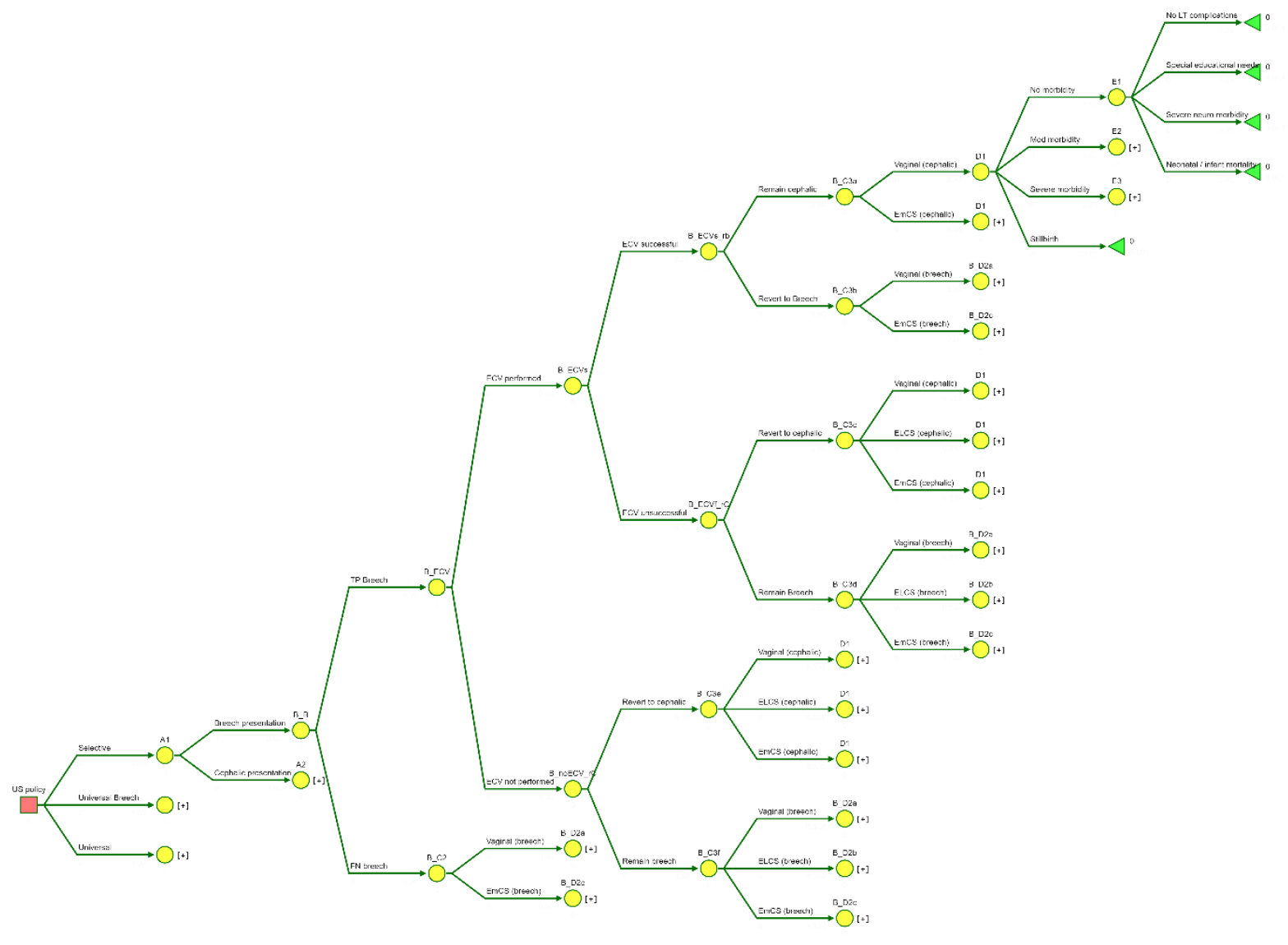

$[+]=$ collapsed sub-branches. Nodes with the same letter have identical subsequent structures, whilst a different number and lowercase letter indicates different probabilities assigned to the next subbranch. The prefix before the underscore indicates a set of probabilities relevant to breech (B_). US = ultrasound; $T P=$ true positive; $F N=$ false negative; $F P=$ false positive; $T N=$ true negative; $E C V=$ external cephalic version; ELCS = Elective Caesarean section; EmCS = Emergency Caesarean section; Exp = Expectant management; $I o L=$ Induction of labour 
Figure A1.2. Outcomes associated with LGA.

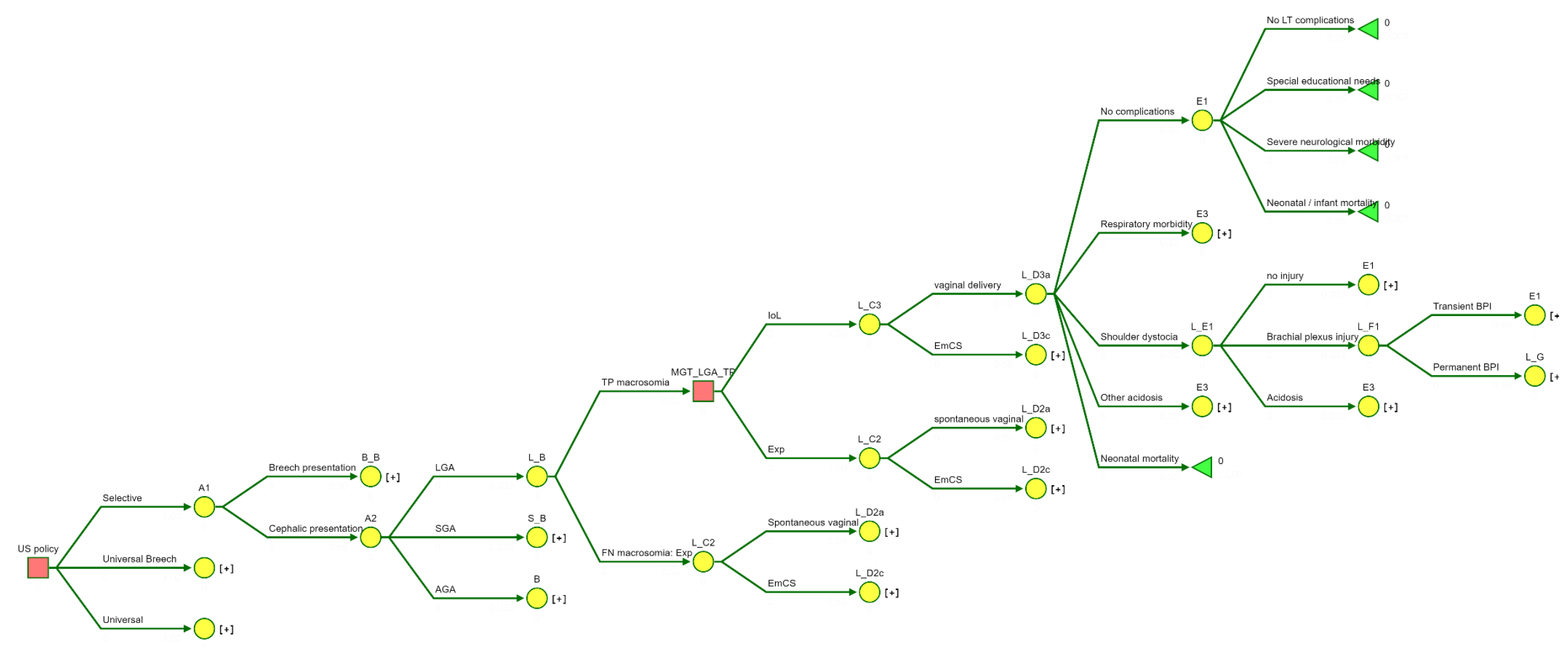

$[+]=$ collapsed sub-branches. Nodes with the same letter have identical subsequent structures, whilst a different number and lowercase letter indicates different probabilities assigned to the next subbranch. The prefix before the underscore indicates a set of probabilities relevant to LGA (L_). US = ultrasound; $T P=$ true positive; $F N=$ false negative; $F P=$ false positive; $T N=$ true negative; EmCS = Emergency Caesarean section; Exp = Expectant management; loL = Induction of labour 
Figure A1.3. Outcomes associated with SGA.

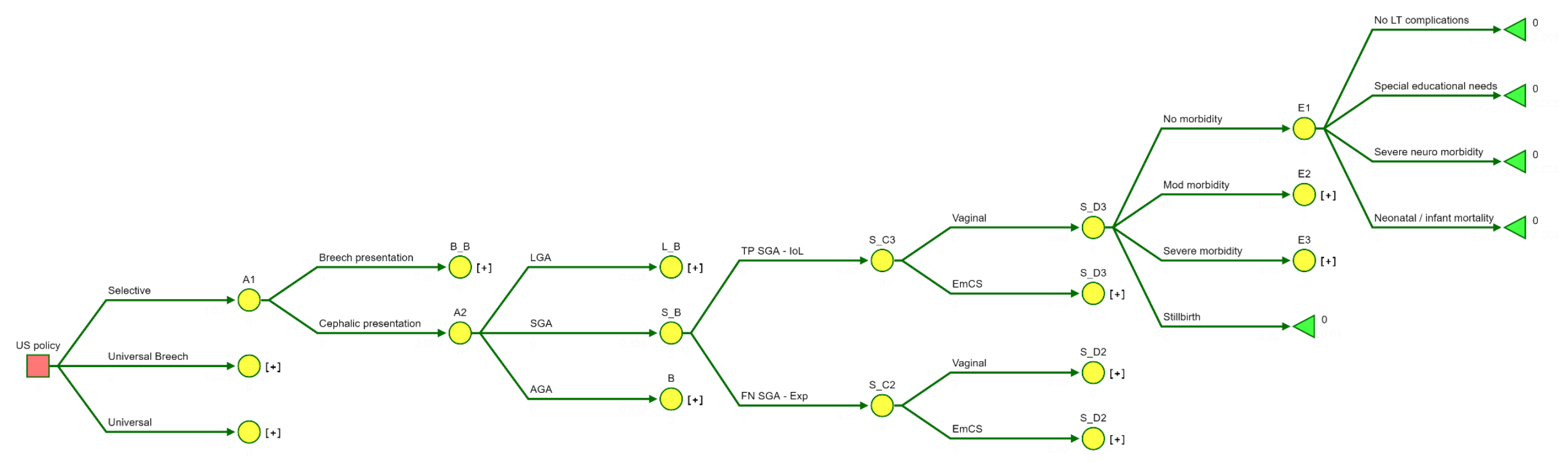

$[+]=$ collapsed sub-branches. Nodes with the same letter have identical subsequent structures, whilst a different number and lowercase letter indicates different probabilities assigned to the next subbranch. The prefix before the underscore indicates a set of probabilities relevant to SGA (S_). US = ultrasound; $T P=$ true positive; $F N=$ false negative; $F P=$ false positive; $T N=$ true negative; EmCS = Emergency Caesarean section; Exp = Expectant management; loL = Induction of labour 


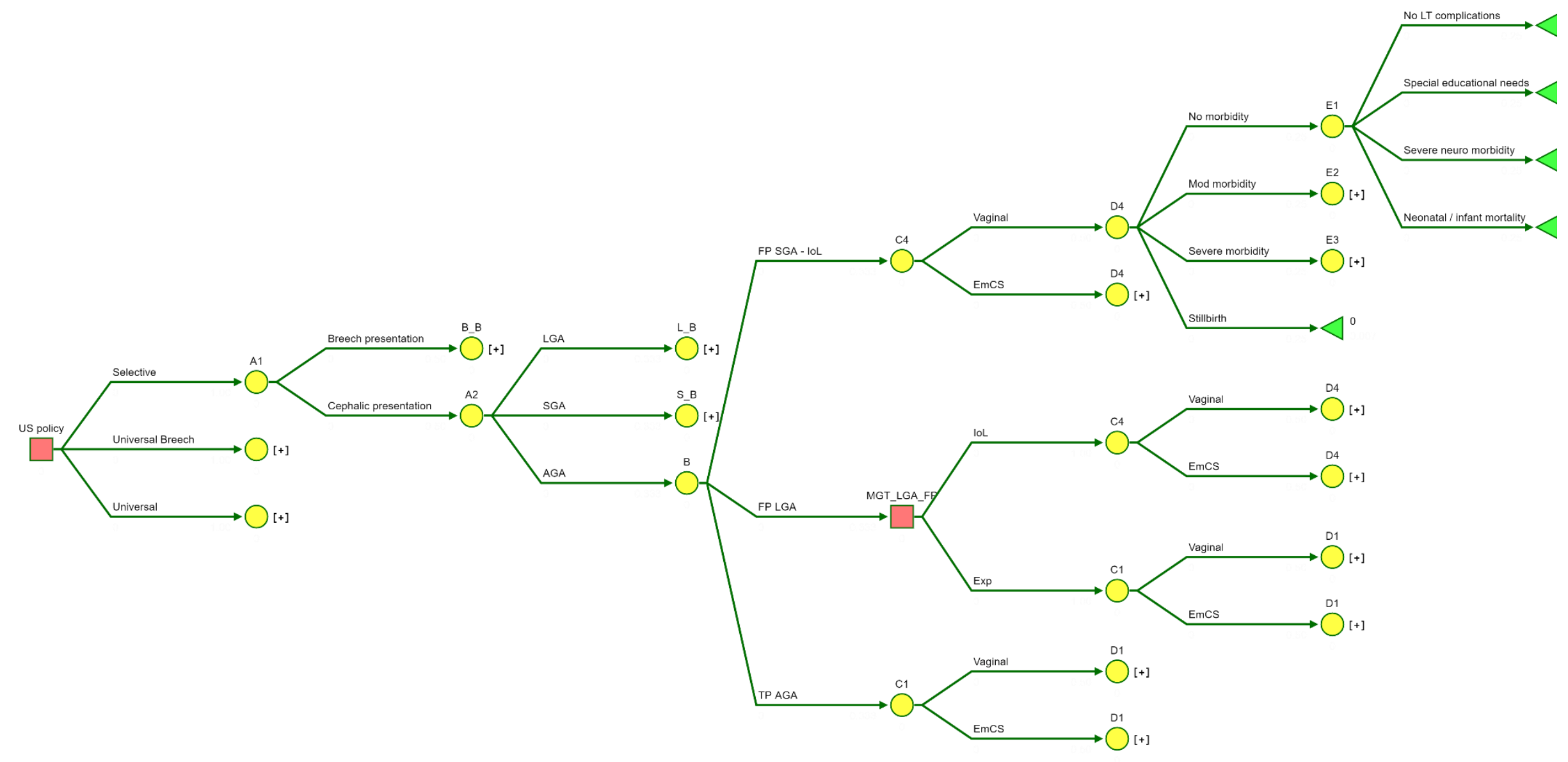

$[+]=$ collapsed sub-branches. Nodes with the same letter have identical subsequent structures, whilst a different number and lowercase letter indicates different probabilities assigned to the next subbranch. US = ultrasound; $T P=$ true positive; $F N=$ false negative; $F P=$ false positive; $T N=$ true negative; EmCS = Emergency Caesarean section; Exp = Expectant management; $I o L=I n d u c t i o n$ of labour 
Appendix 2: Model parameter input values

Summary of model input values: probabilities and costs

Table A2.1 Model inputs for probabilities.

\begin{tabular}{|c|c|c|c|c|c|c|}
\hline Parameter & Mean & $95 \% \mathrm{Cl}$ & Probability distribution & Node & Source & $\begin{array}{l}\text { Quality of } \\
\text { evidence }\end{array}$ \\
\hline \multicolumn{7}{|l|}{ Diagnostic Performance } \\
\hline Prevalence of breech & $4.60 \%$ & $3.98 \%, 5.30 \%$ & $\sim \mathrm{B}(179,3700)$ & $\mathrm{A} 1$ & Wastlund et al. (2019) ${ }^{14}$ & $\mathrm{H}$ \\
\hline Prevalence of LGA & $10.00 \%$ & $10 \%, 10 \%$ & N/A & $\mathrm{A} 2$ & By definition & $\mathrm{H}$ \\
\hline Prevalence of SGA & $10.00 \%$ & $10 \%, 10 \%$ & N/A & $\mathrm{A} 2$ & By definition & $\mathrm{H}$ \\
\hline \multicolumn{7}{|l|}{ Selective US } \\
\hline Specificity SGA - Selective US & $98.10 \%$ & $97.63 \%, 98.52 \%$ & $\sim \mathrm{B}(3556,69)$ & B & Sovio et al. $(2015)^{12}$ & $\mathrm{H}$ \\
\hline Specificity LGA - Selective US & $98.67 \%$ & $98.28 \%, 99.02 \%$ & $\sim B(3640,49)$ & B & Sovio et al. $(2018)^{13}$ & $\mathrm{H}$ \\
\hline Sensitivity SGA - Selective US & $19.60 \%$ & $15.63 \%, 23.90 \%$ & $\sim B(69,283)$ & S_B & Sovio et al. (2015) ${ }^{12}$ & $\mathrm{H}$ \\
\hline Sensitivity LGA - Selective US & $26.55 \%$ & $20.33 \%, 33.28 \%$ & $\sim B(47,130)$ & L_B & Sovio et al. (2018) ${ }^{13}$ & $\mathrm{H}$ \\
\hline Sensitivity breech - selective US & $45.10 \%$ & $37.85 \%, 52.54 \%$ & $\sim \mathrm{B}(79,96)$ & B_B & Wastlund et al. (2019) ${ }^{14}$ & $\mathrm{H}$ \\
\hline \multicolumn{7}{|l|}{ Universal US for fetal size and presentation } \\
\hline Specificity SGA - Universal US & $89.99 \%$ & $88.99 \%, 90.94 \%$ & $\sim \mathrm{B}(3262,363)$ & B & Sovio et al. (2015) ${ }^{12}$ & $\mathrm{H}$ \\
\hline Specificity LGA - Universal US & $96.56 \%$ & $95.95 \%, 97.12 \%$ & $\sim \mathrm{B}(3562,127)$ & B & Sovio et al. (2018) ${ }^{13}$ & $\mathrm{H}$ \\
\hline Sensitivity SGA - Universal US & $56.53 \%$ & $52.33 \%, 61.67 \%$ & $\sim \mathrm{B}(199,153)$ & S_B & Sovio et al. (2015) ${ }^{12}$ & $\mathrm{H}$ \\
\hline Sensitivity LGA - Universal US & $37.85 \%$ & $30.87 \%, 45.10 \%$ & $\sim B(67,110)$ & L_B & Sovio et al. (2018) ${ }^{13}$ & $\mathrm{H}$ \\
\hline Sensitivity breech - Universal US & $100 \%$ & $100 \%, 100 \%$ & N/A & B_B & Assumption & N/A \\
\hline \multicolumn{7}{|l|}{ Universal US for fetal presentation only } \\
\hline Specificity SGA - Presentation-only scan & $98.10 \%$ & $97.63 \%, 98.52 \%$ & $\sim \mathrm{B}(3556,69)$ & B & Sovio et al. (2015) ${ }^{12}$ & $\mathrm{H}$ \\
\hline Specificity LGA - Presentation-only scan & $98.67 \%$ & $98.28 \%, 99.02 \%$ & $\sim B(3640,49)$ & B & Sovio et al. (2018) ${ }^{13}$ & $\mathrm{H}$ \\
\hline Sensitivity SGA - Presentation-only scan & $19.60 \%$ & $15.63 \%, 23.90 \%$ & $\sim \mathrm{B}(69,283)$ & S_B & Sovio et al. (2015) ${ }^{12}$ & $\mathrm{H}$ \\
\hline Sensitivity LGA - Presentation-only scan & $26.55 \%$ & $20.33 \%, 33.28 \%$ & $\sim \mathrm{B}(47,130)$ & L_B & Sovio et al. (2018) ${ }^{13}$ & $\mathrm{H}$ \\
\hline Sensitivity breech - Presentation-only scan & $100 \%$ & $100 \%, 100 \%$ & N/A & B_B & Assumption & N/A \\
\hline \multicolumn{7}{|l|}{ Mode of delivery } \\
\hline EmCS delivery | AGA and Exp Mgt & $20.70 \%$ & $19.4 \%, 22.06 \%$ & $\sim \mathrm{B}(735,2813)$ & $\mathrm{C} 1$ & Wastlund et al. ${ }^{14}$ & $\mathrm{H}$ \\
\hline
\end{tabular}




\begin{tabular}{|c|c|c|c|c|c|c|}
\hline Parameter & Mean & $95 \% \mathrm{Cl}$ & Probability distribution & Node & Source & $\begin{array}{l}\text { Quality of } \\
\text { evidence }\end{array}$ \\
\hline RR EmCS delivery I SGA and Exp Mgt [FN] vs. C1 & 1.9 & $1.4,2.5$ & $\sim \operatorname{LN}(0.642,0.14)$ & S_C2 & Monier et al. ${ }^{21}$ & M \\
\hline RR EMCS | induced, SGA [TP] vs. C1 & 2.9 & $1.8,4.7$ & $\sim \operatorname{LN}(1.065,0.246)$ & S_C3 & Monier et al. ${ }^{21}$ & L \\
\hline RR EMCS | induced, AGA, [FP SGA] vs. C1 & 0.84 & $0.76,0.93$ & $\sim \operatorname{LN}(-0.174,0.052)$ & $\mathrm{C} 4$ & Grobman et al. ${ }^{22}$ & $\mathrm{H}$ \\
\hline OR of EmCS delivery | LGA and Exp Mgt [FN] vs. C1 & 1.792 & $0.718,4.471$ & $\sim \operatorname{LN}(0.583,0.466)$ & L_C2 & Blackwell et al..$^{23}$ & M \\
\hline OR of EmCS delivery | LGA and Induce [TP] vs. L_C2 & 0.92 & $0.85,0.99$ & $\sim \operatorname{LN}(-0.083,0.037)$ & L_C3 & Middleton et al. ${ }^{24}$ & L \\
\hline EmCS delivery | Breech and Exp Mgt [FN] & $57.69 \%$ & $38.67 \%, 75.62 \%$ & $\sim \mathrm{B}(15,11)$ & B_C2 & Leung et al. ${ }^{25}$ & M \\
\hline EmCS delivery | breech, ECV success, remain cephalic & $27.27 \%$ & $6.69 \%, 55.64 \%$ & $\sim \mathrm{B}(3,8)$ & B_C3a & Wastlund et al. ${ }^{14}$ & $\mathrm{H}$ \\
\hline EmCS delivery | breech, ECV success, revert breech & $57.69 \%$ & $38.67 \%, 75.62 \%$ & $\sim \mathrm{B}(15,11)$ & B_C3b & Leung et al. ${ }^{25}$ & M \\
\hline Vaginal delivery | breech, ECV fail, revert cephalic & $52.38 \%$ & $31.51 \%, 72.80 \%$ & $\sim D(11,1,9)$ & B_C3c & Wastlund et al. ${ }^{14}$ & $\mathrm{H}$ \\
\hline ELCS delivery | breech, ECV fail, revert cephalic & $4.76 \%$ & $0.13 \%, 16.84 \%$ & - & B_C3c & Wastlund et al. ${ }^{14}$ & \\
\hline EmCS delivery | breech, ECV fail, revert cephalic & $42.86 \%$ & $23.07 \%, 63.97 \%$ & - & B_C3c & Wastlund et al. ${ }^{14}$ & \\
\hline Vaginal delivery | breech, ECV fail, remain breech & $0 \%$ & $0 \%, 0 \%$ & $\sim D(0,54,18)$ & B_C3d & Wastlund et al. ${ }^{14}$ & $\mathrm{H}$ \\
\hline ELCS delivery | breech, ECV fail, remain breech & $75 \%$ & $64.47 \%, 84.22 \%$ & - & B_C3d & Wastlund et al. ${ }^{14}$ & \\
\hline EmCS delivery | breech, ECV fail, remain breech & $25 \%$ & $15.78 \%, 35.53 \%$ & - & B_C3d & Wastlund et al. ${ }^{14}$ & \\
\hline Vaginal delivery | breech, no ECV, revert cephalic & $52.38 \%$ & $31.51 \%, 72.80 \%$ & $\sim D(11,1,9)$ & B_C3e & Wastlund et al. ${ }^{14}$ & $\mathrm{H}$ \\
\hline ELCS delivery | breech, no ECV, revert cephalic & $4.76 \%$ & $0.13 \%, 16.84 \%$ & - & B_C3e & Wastlund et al. ${ }^{14}$ & \\
\hline EmCS delivery | breech, no ECV, revert cephalic & $42.86 \%$ & $23.07 \%, 63.97 \%$ & - & B_C3e & Wastlund et al. ${ }^{14}$ & \\
\hline Vaginal delivery | breech, no ECV, remain breech & $0 \%$ & $0 \%, 0 \%$ & $\sim D(0,52,20)$ & B_C3f & Wastlund et al. ${ }^{14}$ & $\mathrm{H}$ \\
\hline ELCS delivery | breech, no ECV, remain breech & $72.22 \%$ & $61.38 \%, 81.88 \%$ & - & B_C3f & Wastlund et al. ${ }^{14}$ & \\
\hline EmCS delivery | breech, no ECV, remain breech & $27.77 \%$ & $18.12 \%, 38.62 \%$ & - & B_C3f & Wastlund et al. ${ }^{14}$ & \\
\hline \multicolumn{7}{|l|}{ External cephalic version } \\
\hline ECV attempted & $47.46 \%$ & $40.16 \%, 54.81 \%$ & $\sim \mathrm{B}(84,93)$ & B_ECV & Wastlund et al. ${ }^{14}$ & $\mathrm{H}$ \\
\hline ECV not attempted, spontaneous reversion to cephalic & $22.58 \%$ & $14.72 \%, 31.56 \%$ & $\sim \mathrm{B}(21,72)$ & B_noECV_rc & Wastlund et al. ${ }^{14}$ & $\mathrm{H}$ \\
\hline Probability ECV successful & $14.29 \%$ & $7.70 \%, 22.48 \%$ & $\sim \mathrm{B}(12,72)$ & B_ECVs & Wastlund et al. ${ }^{14}$ & $\mathrm{H}$ \\
\hline Probability of reverting to breech post successful ECV & $8.33 \%$ & $0.23 \%, 28.49 \%$ & $\sim \mathrm{B}(1,11)$ & B_ECVs_rb & Wastlund et al. ${ }^{14}$ & $\mathrm{H}$ \\
\hline Probability of spontaneous reversion to cephalic post ECV failure & $2.31 \%$ & $0.48 \%, 5.49 \%$ & $\sim \mathrm{B}(3,127)$ & B_ECVf_rc & Ben-Meir et al. ${ }^{26}$ & $\mathrm{H}$ \\
\hline \multicolumn{7}{|l|}{ Outcomes for LGA model } \\
\hline Respiratory morbidity, baseline & $0.32 \%$ & $0.20 \%, 0.46 \%$ & $\sim B(22,6933)$ & - & Morrison et al. ${ }^{27}$ & $\mathrm{H}$ \\
\hline
\end{tabular}




\begin{tabular}{|c|c|c|c|c|c|c|}
\hline Parameter & Mean & $95 \% \mathrm{Cl}$ & Probability distribution & Node & Source & $\begin{array}{l}\text { Quality of } \\
\text { evidence }\end{array}$ \\
\hline Shoulder dystocia, baseline & $0.63 \%$ & $0.60 \%, 0.66 \%$ & $\sim B(1686,265542)$ & - & Ouzounian et al. ${ }^{28}$ & M \\
\hline Other acidosis, baseline & $0.68 \%$ & $0.22 \%, 1.40 \%$ & $\sim \mathrm{B}(5,726)$ & - & Middleton et al. ${ }^{24}$ & $\mathrm{H}$ \\
\hline Perinatal mortality, baseline & $0.155 \%$ & $0.145 \%, 0.165 \%$ & $\sim B(984,634412)$ & - & Moraitis et al. ${ }^{29}$ & M \\
\hline RR respiratory morbidity, LGA vs. AGA [FN \& ExpMan LGA policy] & 0.75 & $0.5125,0.9875$ & $\sim U(0.5,1)$ & L_D2a & Expert opinion & L \\
\hline OR shoulder dystocia, LGA vs. AGA [FN \& ExpMan LGA policy] & 7.18 & $2.06,25.00$ & $\sim \operatorname{LN}(1.971,0.637)$ & L_D2a & Rossi et al. ${ }^{30}$ & $\mathrm{H}$ \\
\hline OR other acidosis, LGA vs. AGA [FN \& ExpMan LGA policy] & 2.88 & $1.34,6.22$ & $\sim \operatorname{LN}(1.058,0.393)$ & L_D2a & Rossi et al. ${ }^{30}$ & M \\
\hline OR perinatal mortality, LGA vs. AGA [FN \& ExpMan LGA policy] & 1.77 & $0.30,10.34$ & $\sim \operatorname{LN}(0.571,0.901)$ & L_D2a & Rossi et al. ${ }^{30}$ & M \\
\hline OR respiratory morbidity, LGA vs. AGA, EMCS [FN \& ExpMan LGA policy] & 5.33 & $3.50,7.40$ & $\sim \operatorname{LN}(1.674,0.167)$ & L_D2C & Morrison et al. ${ }^{27}$ & $\mathrm{H}$ \\
\hline P shoulder dystocia, LGA, EMCS [FN \& ExpMan LGA policy] & 0 & 0,0 & N/A & L_D2c & Assumption & $\mathrm{H}$ \\
\hline OR other acidosis, LGA, EMCS [FN \& ExpMan LGA policy] & 1.867 & $1.217,2.865$ & $\sim \operatorname{LN}(0.625,0.218)$ & L_D2c & Chongsuvivatwong et al. ${ }^{31}$ & M \\
\hline OR perinatal mortality, LGA, EMCS [FN \& ExpMan LGA policy] & 1.781 & $1.266,2.505$ & $\sim \operatorname{LN}(0.577,0.174)$ & L_D2c & Chongsuvivatwong et al. ${ }^{31}$ & M \\
\hline OR respiratory morbidity, LGA, Induction of labour, vaginal delivery [TP] & 0.54 & $0.373,0.783$ & $\sim \operatorname{LN}(-0.616,0.19)$ & L_D3a & Gibson et al. ${ }^{32}$ & M \\
\hline RR shoulder dystocia, LGA, Induction of labour, vaginal delivery [TP] & 0.6 & $0.37,0.98$ & $\sim \operatorname{LN}(-0.511,0.25)$ & L_D3a & Boulvain et al. ${ }^{33}$ & M \\
\hline RR acidosis, LGA, Induction of labour, vaginal delivery [TP] & 1.66 & $0.61,4.55$ & $\sim \operatorname{LN}(0.507,0.514)$ & L_D3a & Middleton et al. ${ }^{24}$ & M \\
\hline RR perinatal mortality, LGA, Induction of labour, vaginal delivery [TP] & 0.33 & $0.14,0.78$ & $\sim \operatorname{LN}(-1.109,0.439)$ & L_D3a & Middleton et al. ${ }^{24}$ & M \\
\hline OR respiratory morbidity, LGA, Induction of labour, EmCS [TP] & 0.54 & $0.373,0.783$ & $\sim \operatorname{LN}(-0.616,0.19)$ & L_D3c & Gibson et al. 32 & M \\
\hline P shoulder dystocia, LGA, Induction of labour, EmCS [TP] & 0 & 0,0 & N/A & L_D3c & Assumption & $\mathrm{H}$ \\
\hline RR acidosis, LGA, Induction of labour, EmCS [TP] & 1.66 & $0.61,4.55$ & $\sim \operatorname{LN}(0.507,0.514)$ & L_D3c & Middleton et al. ${ }^{24}$ & M \\
\hline RR perinatal mortality, LGA, Induction of labour, EmCS [TP] & 0.33 & $0.14,0.78$ & $\sim \operatorname{LN}(-1.109,0.439)$ & L_D3c & Middleton et al. ${ }^{24}$ & M \\
\hline Risk of acidosis | shoulder dystocia & 0.07 & $0.0630,0.1112$ & $\sim B(36,478)$ & L_E1 & MacKenzie et al. ${ }^{34}$ & L \\
\hline Risk of BPI | shoulder dystocia & 0.0856 & $0.0496,0.0936$ & $\sim B(44,470)$ & L_E1 & Mackenzie et al. $34 \mathrm{c}$ & L \\
\hline Risk of permanent BPI & 0.055 & $0.024,0.098$ & $\sim \mathrm{B}(8,137)$ & L_F1 & Sandmire et al. ${ }^{35 \mathrm{c}}$ & M \\
\hline \multicolumn{7}{|l|}{ Neonatal morbidity } \\
\hline Risk of moderate neonatal morbidity (AGA) [FP] & $5.62 \%$ & $0.0488,0.0641$ & $\sim \mathrm{B}(198,3325)$ & D1 & The POP study ${ }^{c}$ & $\mathrm{H}$ \\
\hline Risk of severe neonatal morbidity (AGA) [FP] & $0.62 \%$ & $0.0039,0.0091$ & $\sim \mathrm{B}(22,3501)$ & D1 & The POP study ${ }^{c}$ & $\mathrm{H}$ \\
\hline Risk of perinatal death (AGA) [FP] & $0.155 \%$ & $0.145 \%, 0.165 \%$ & $\sim B(984,634412)$ & D1 & Moraitis et al. ${ }^{29}$ & M \\
\hline OR moderate neonatal morbidity (SGA vs. AGA, ExpMan) & 2.48 & $1.75,3.51$ & $\sim \operatorname{LN}(0.91,0.18)$ & S_D2 & The POP Study ${ }^{c}$ & $\mathrm{H}$ \\
\hline OR severe neonatal morbidity (SGA vs. AGA, ExpMan) & 1.88 & $0.65,5.50$ & $\sim \operatorname{LN}(0.63,0.55)$ & S_D2 & The POP Study ${ }^{c}$ & $\mathrm{H}$ \\
\hline
\end{tabular}




\begin{tabular}{|c|c|c|c|c|c|c|}
\hline Parameter & Mean & $95 \% \mathrm{Cl}$ & Probability distribution & Node & Source & $\begin{array}{l}\text { Quality of } \\
\text { evidence }\end{array}$ \\
\hline OR perinatal death (SGA vs. AGA, ExpMan) & 4.39 & $3.84,5.03$ & $\sim \operatorname{LN}(1.48,0.07)$ & S_D2 & Moraitis et al. ${ }^{29}$ & $\mathrm{H}$ \\
\hline RR moderate morbidity | induce SGA vs. not inducing SGA [TP] & 0.7 & $0.50,0.98$ & $\sim \operatorname{LN}(-0.357,0.172)$ & S_D3 & Middleton et al. ${ }^{24}$ & $\mathrm{~L}$ \\
\hline RR severe morbidity | induce SGA vs. not inducing SGA [TP] & 0.7 & $0.50,0.98$ & $\sim \operatorname{LN}(-0.357,0.172)$ & S_D3 & Middleton et al. ${ }^{24}$ & $\mathrm{~L}$ \\
\hline RR perinatal death | induce SGA vs. not inducing SGA [TP] & 0.33 & $0.11,0.96$ & $\sim \operatorname{LN}(-1.109,0.553)$ & S_D3 & Middleton et al. ${ }^{24}$ & $\mathrm{~L}$ \\
\hline OR of moderate neonatal morbidity if induce / AGA [FP SGA or LGA] & 1.92 & $1.71,2.15$ & $\sim \operatorname{LN}(0.652,0.058)$ & D4 & Stock et al. ${ }^{36}$ & $\mathrm{H}$ \\
\hline OR of severe neonatal morbidity if induce | AGA [FP SGA or LGA] & 1.92 & $1.71,2.15$ & $\sim \operatorname{LN}(0.652,0.058)$ & D4 & Stock et al. ${ }^{36}$ & $\mathrm{H}$ \\
\hline OR of perinatal death if induce I AGA [FP SGA or LGA] & 0.15 & $0.03,0.68$ & $\sim \operatorname{LN}(-1.897,0.771)$ & D4 & Stock et al. ${ }^{36}$ & $\mathrm{H}$ \\
\hline $\begin{array}{l}\text { OR of moderate neonatal morbidity I } \\
\text { vaginal breech vs. vaginal cephalic delivery }\end{array}$ & 6.70 & $5.9,7.6$ & $\sim \operatorname{LN}(1.902,0.064)$ & B_D2a & Thorngren-Jerneck et al. ${ }^{37}$ & $\mathrm{H}$ \\
\hline $\begin{array}{l}\text { OR of severe neonatal morbidity I } \\
\text { vaginal breech vs. vaginal cephalic delivery }\end{array}$ & 6.70 & $5.9,7.6$ & $\sim \operatorname{LN}(1.902,0.064)$ & B_D2a & Thorngren-Jerneck et al. ${ }^{37}$ & $\mathrm{H}$ \\
\hline $\begin{array}{l}\text { OR of perinatal death I } \\
\text { vaginal breech vs. vaginal cephalic delivery }\end{array}$ & 6.68 & $2.75,16.22$ & $\sim \operatorname{LN}(1.899,0.453)$ & B_D2a & Moraitis et al. ${ }^{29}$ & $\mathrm{H}$ \\
\hline RR of moderate morbidity | ELCS vs. vaginal breech delivery & 0.43 & $0.12,1.47$ & $\sim \operatorname{LN}(-0.844,0.627)$ & B_D2b & Hofmeyr et al. ${ }^{38}$ & $\mathrm{H}$ \\
\hline RR of severe morbidity | ELCS vs. vaginal breech delivery & 0.11 & $0.01,0.87$ & $\sim \operatorname{LN}(-2.207,1.055)$ & B_D2b & Hofmeyr et al. ${ }^{38}$ & $\mathrm{H}$ \\
\hline RR of perinatal death | ELCS vs. vaginal breech delivery & 0.29 & $0.1,0.86$ & $\sim \operatorname{LN}(-1.238,0.555)$ & B_D2b & Hofmeyr et al. ${ }^{38}$ & H \\
\hline OR of moderate morbidity | EmCS vs. vaginal breech delivery & 0.533 & $0.192,1.482$ & $\sim \operatorname{LN}(-0.629,0.522)$ & B_D2c & Pasupathy et al. $39 \mathrm{c}$ & M \\
\hline OR of severe morbidity | EmCS vs. vaginal breech delivery & 0.533 & $0.192,1.482$ & $\sim \operatorname{LN}(-0.629,0.522)$ & B_D2c & Pasupathy et al. ${ }^{39} \mathrm{c}$ & M \\
\hline OR of perinatal death | EmCS vs. vaginal breech delivery & 0.533 & $0.192,1.482$ & $\sim \operatorname{LN}(-0.629,0.522)$ & B_D2c & Pasupathy et al. $39 \mathrm{c}$ & M \\
\hline \multicolumn{7}{|l|}{ Risk of long-term outcomes from neonatal morbidity } \\
\hline Risk of SEN | no neonatal morbidity & 0.0474 & $0.0467,0.0480$ & $\sim \mathrm{B}(18736,376891)$ & E1 & MacKay et al. ${ }^{40}$ & $\mathrm{H}$ \\
\hline Risk of neurological morbidity | no neonatal morbidity & 0.0008 & $0.0007,0.0008$ & $\sim \mathrm{B}(906,1193647)$ & E1 & Persson et al. ${ }^{41}$ & $\mathrm{H}$ \\
\hline Risk of neonatal/infant mortality | no neonatal morbidity & 0.002 & $0.0020,0.0021$ & $\sim \mathrm{B}(2074,1011289)$ & E1 & Iliodromiti et al. ${ }^{42}$ & $\mathrm{H}$ \\
\hline OR of SEN | moderate neonatal morbidity & 1.55 & $1.43,1.67$ & $\sim \operatorname{LN}(0.438,0.038)$ & E2 & MacKay et al. ${ }^{40}$ & $\mathrm{H}$ \\
\hline RR of neurological morbidity | moderate neonatal morbidity & 10.4 & $7.8,13.9$ & $\sim \operatorname{LN}(2.34,0.149)$ & E2 & Persson et al. ${ }^{41}$ & H \\
\hline RR of neonatal/infant mortality | moderate morbidity & 12.82 & $9.33,17.61$ & $\sim \operatorname{LN}(2.551,0.162)$ & E2 & Iliodromiti et al..$^{42}$ & $\mathrm{H}$ \\
\hline OR of SEN | severe neonatal morbidity & 1.66 & $1.46,1.88$ & $\sim \operatorname{LN}(0.507,0.063)$ & E3 & MacKay et al. ${ }^{40}$ & $\mathrm{H}$ \\
\hline RR of neurological morbidity | severe morbidity & 145.5 & 104.0, 204.1 & $\sim \operatorname{LN}(4.98,0.173)$ & E3 & Persson et al. ${ }^{41}$ & H \\
\hline RR of neonatal/infant mortality | severe morbidity & 60.61 & $48.17,76.26$ & $\sim \operatorname{LN}(4.104,0.117)$ & E3 & Iliodromiti et al. ${ }^{42}$ & $\mathrm{H}$ \\
\hline
\end{tabular}




\begin{tabular}{|c|c|c|c|c|c|c|}
\hline Parameter & Mean & $95 \% \mathrm{Cl}$ & Probability distribution & Node & Source & $\begin{array}{l}\text { Quality of } \\
\text { evidence }\end{array}$ \\
\hline \multicolumn{7}{|l|}{ Unit costs and related probabilities } \\
\hline Ultrasound scan & £107.06 & $f 70.98,134.92$ & $\sim G(4.9604,22.8062)$ & A & NHS reference costs $2016-17^{43 c}$ & H \\
\hline Presentation-only scan & $£ 48.71$ & $£ 8.96,88.46$ & $\sim U(6.87,90.55)$ & A & Expert opinion & $\mathrm{N} / \mathrm{A}$ \\
\hline Proportion scanned with US (selective screening) & 0.3499 & $0.3349,0.3650$ & $\sim \mathrm{B}(1351,2510)$ & A & Sovio et al. ${ }^{12}$ & $\mathrm{H}$ \\
\hline Induction of labour (difference vs. normal delivery) & $£ 125$ & $-£ 1343,1594$ & $\sim N(125.3,749.2)$ & B1, B2 & Vijgen et al. ${ }^{44}$ & M \\
\hline Cost of vaginal (cephalic) delivery & $£ 1,834$ & f1750, 2236 & $\sim G(7.2606,252.5824)$ & $\mathrm{C} 1-\mathrm{C} 4$ & NHS reference costs $2016-17^{43 c}$ & $\mathrm{H}$ \\
\hline Relative cost difference (vaginal breech vs. cephalic delivery) & 1.1633 & $1.0982,1.2284$ & $\sim N(1.1633,0.0332)$ & $\begin{array}{l}\text { B_C3b, B_C3d, } \\
\text { B_C3f, B_C2, }\end{array}$ & Palencia et al. ${ }^{45}$ & M \\
\hline Cost of ECV & $£ 292.30$ & $£ 287.5,297.1$ & $\sim U(287.22,297.38)$ & B_ECV & James et al. $46 \mathrm{c}$ & M \\
\hline Cost of emergency Caesarean section & $£ 4,688$ & $£ 3816,5443$ & $\sim G(14.7329,318.1354)$ & $\mathrm{C} 1-\mathrm{C} 4$ & NHS reference costs $2016-17^{43 c}$ & $\mathrm{H}$ \\
\hline Cost of elective Caesarean section & $£ 3,412$ & $f 2680,4038$ & $\sim G(11.1212,307.0169)$ & $\mathrm{C} 1-\mathrm{C} 4$ & NHS reference costs $2016-17^{43 c}$ & $\mathrm{H}$ \\
\hline Cost of Special Care Baby Unit admission & $£ 1,064$ & $£ 487,1862$ & $\sim \mathrm{G}(9.0371,117.7307)$ & D1 - D4 & NHS reference costs $2016-17^{43 c}$ & H \\
\hline Cost of Neonatal High Dependency Unit admission & $£ 1,346$ & $£ 807,2020$ & $\sim G(18.7696,71.7047)$ & D1 - D4 & NHS reference costs $2016-17^{43 c}$ & $\mathrm{H}$ \\
\hline Cost of Neonatal Intensive Care Unit admission & $f 2,590$ & $£ 1280,4352$ & $\sim G(10.7403,241.0768)$ & D1 - D4 & NHS reference costs $2016-17^{43 c}$ & H \\
\hline Proportion of neonates admitted to SCBU & $74 \%$ & $65 \%, 82 \%$ & $\sim D(74,7,19)$ & D1 - D4 & Alfirevic et al. ${ }^{47}$ & M \\
\hline Proportion of neonates admitted to NHDU & $7 \%$ & $3 \%, 13 \%$ & - & D1 - D4 & Alfirevic et al. ${ }^{47}$ & \\
\hline Proportion of neonates admitted to NICU & $19 \%$ & $12 \%, 27 \%$ & - & D1 - D4 & Alfirevic et al. ${ }^{47}$ & \\
\hline $\begin{array}{l}\text { Probability of admission to care I } \\
\text { no neonatal morbidity }\end{array}$ & 0.074 & $0.066,0.082$ & $\sim B(292,3659)$ & D1 - D4 & Sovio et al. ${ }^{12}$ & $\mathrm{H}$ \\
\hline $\begin{array}{l}\text { Odds ratio of admission to care } \\
\text { Moderate neonatal morbidity }\end{array}$ & 11.29 & $5.90,21.60$ & $\sim \operatorname{LN}(2.424,0.331)$ & D1 - D4 & Sovio et al. ${ }^{12}$ & $\mathrm{H}$ \\
\hline $\begin{array}{l}\text { Probability of admission to care | } \\
\text { Severe neonatal morbidity }\end{array}$ & 1 & 1,1 & N/A & D1 - D4 & Assumption & N/A \\
\hline Short-term cost of acidosis / anoxia & $£ 3,240$ & $£ 806,7328$ & $\sim G(3.6143,895.6169)$ & L_E1, L_D2a & Own estimation ${ }^{c}$ & L \\
\hline Short-term cost of respiratory morbidity & $f 2,011$ & f993, 3381 & $\sim G(10.7125,187.6316)$ & L_D2a, L_D3a & Own estimation ${ }^{c}$ & $\mathrm{~L}$ \\
\hline Cost of transient BPI & $£ 2,066$ & $f 1033,4132$ & $\sim \operatorname{LN}(7.6334,0.3536)$ & L_F1 & Culligan et al. ${ }^{48}$ & M \\
\hline Cost of permanent BPI & $£ 14,134$ & $f 7068,28264$ & $\sim \operatorname{LN}(9.5563,0.03536)$ & L_F1 & Culligan et a. ${ }^{48 \mathrm{c}}$ & M \\
\hline Cost of perinatal or infant mortality & $£ 1,664$ & $£ 1372,1956$ & $\sim U(1357,1971)$ & $\mathrm{D} 1 \& \mathrm{E} 1-3$ & Mistry et al. ${ }^{49}$ & M \\
\hline
\end{tabular}




\begin{tabular}{|c|c|c|c|c|c|c|}
\hline Parameter & Mean & $95 \% \mathrm{Cl}$ & Probability distribution & Node & Source & $\begin{array}{l}\text { Quality of } \\
\text { evidence }\end{array}$ \\
\hline Special educational needs (per annum) & $f 7,428$ & $£ 4467,10389$ & $\sim N(7428.1,1511)$ & $\mathrm{E} 1-\mathrm{E} 3$ & Barrett et al. ${ }^{50}$ & M \\
\hline Severe neurological morbidity (per annum) & $£ 2,930$ & $£ 1465,5859$ & $\sim \operatorname{LN}(7.9826,0.3536)$ & $\mathrm{E} 1-\mathrm{E} 3$ & Access economics ${ }^{51}$ & M \\
\hline Utility associated with permanent brachial plexus injury & 0.5 & $0.31,0.69$ & $\sim U(0.3,0.7)$ & L_G & Culligan et al. ${ }^{48}$ & M \\
\hline Disutility associated with SEN & 0 & & $\mathrm{n} / \mathrm{a}$ & $E 1-E 3$ & Assumption & L \\
\hline$\%$ GMFCS 1 (mild) & $22.2 \%$ & $15.4 \%, 29.8 \%$ & $\sim D(28,15,23,32,28)$ & $\mathrm{E} 1-\mathrm{E} 3$ & Young et al. ${ }^{52}$ & $\mathrm{H}$ \\
\hline \% GMFCS 2 (mild) & $11.9 \%$ & $6.9 \%, 18.1 \%$ & - & $\mathrm{E} 1-\mathrm{E} 3$ & Young et al. ${ }^{52}$ & \\
\hline$\%$ GMFCS 3 (moderate) & $18.3 \%$ & $12.0 \%, 25.4 \%$ & - & $\mathrm{E} 1-\mathrm{E} 3$ & Young et al. ${ }^{52}$ & \\
\hline \% GMFCS 4 (severe) & $25.4 \%$ & $18.2 \%, 33.3 \%$ & - & $\mathrm{E} 1-\mathrm{E} 3$ & Young et al. 52 & \\
\hline \% GMFCS 5 (severe) & $22.2 \%$ & $15.4 \%, 29.8 \%$ & - & $\mathrm{E} 1-\mathrm{E} 3$ & Young et al. ${ }^{52}$ & \\
\hline Utility, age 0 to 24 & 0.94 & $0.926,0.954$ & $\sim N(0.94,0.007)$ & $\mathrm{E} 1-\mathrm{E} 3$ & Szende et al..$^{53}$ & $\mathrm{H}$ \\
\hline Utility, age 25 to 34 & 0.927 & $0.915,0.939$ & $\sim N(0.927,0.006)$ & $\mathrm{E} 1-\mathrm{E} 3$ & Szende et al. ${ }^{53}$ & $\mathrm{H}$ \\
\hline Utility, age 35 to 44 & 0.911 & $0.897,0.925$ & $\sim N(0.911,0.007)$ & $\mathrm{E} 1-\mathrm{E} 3$ & Szende et al. ${ }^{53}$ & $\mathrm{H}$ \\
\hline Utility, age 45 to 54 & 0.847 & $0.825,0.869$ & $\sim N(0.847,0.011)$ & $\mathrm{E} 1-\mathrm{E} 3$ & Szende et al. ${ }^{53}$ & $\mathrm{H}$ \\
\hline Utility, age 55 to 64 & 0.799 & $0.775,0.823$ & $\sim N(0.799,0.012)$ & $E 1-E 3$ & Szende et al. ${ }^{53}$ & $\mathrm{H}$ \\
\hline Utility, age 65 to 74 & 0.779 & $0.755,0.803$ & $\sim N(0.779,0.012)$ & $\mathrm{E} 1-\mathrm{E} 3$ & Szende et al. ${ }^{53}$ & $\mathrm{H}$ \\
\hline Utility, age $75+$ & 0.726 & $0.697,0.755$ & $\sim N(0.726,0.015)$ & $E 1-E 3$ & Szende et al. ${ }^{53}$ & $\mathrm{H}$ \\
\hline Disutility, GMFCS 1 & 0.124 & $0.003,0.466$ & $\sim \mathrm{G}(0.95,0.13)$ & $\mathrm{E} 1-\mathrm{E} 3$ & Leigh et al. ${ }^{54}$ & M \\
\hline Disutility, GMFCS 2 & 0.322 & $0.123,0.614$ & $\sim \mathrm{G}(6.43,0.05)$ & $\mathrm{E} 1-\mathrm{E} 3$ & Leigh et al. ${ }^{54}$ & M \\
\hline Disutility, GMFCS 3 & 0.497 & $0.201,0.924$ & $\sim G(7.1,0.07)$ & $\mathrm{E} 1-\mathrm{E} 3$ & Leigh et al. ${ }^{54}$ & M \\
\hline Disutility, GMFCS 4 & 1.035 & $0.845,1.244$ & $\sim \mathrm{G}(103.5,0.01)$ & $\mathrm{E} 1-\mathrm{E} 3$ & Leigh et al. ${ }^{54}$ & M \\
\hline Disutility, GMFCS 5 & 1.35 & $0.985,1.772$ & $\sim G(45,0.03)$ & $\mathrm{E} 1-\mathrm{E} 3$ & Leigh et al. ${ }^{54}$ & M \\
\hline
\end{tabular}

${ }^{a}$ Distributions: $B=$ Beta,$D=$ Dirichlet $; G=$ Gamma, $L N=$ Log-normal, $N=$ Normal, $U=$ Uniform 
${ }^{b}$ Quality assessment: $\mathrm{H}=$ High - good quality directly relevant evidence (e.g. directly relevant population, well conducted RCT for relative effects, or cohort for baseline effects). $M=$ Medium - directly relevant evidence but poorer quality source (e.g. retrospective cohort for relative treatment effect). $L=L$ Low - lack of direct evidence or informed by expert opinion.

c Parameter estimated based upon data from the source, rather than directly from the source. Details are provided in text below.

$B P I=$ Brachial plexus injury, $E C V=$ External cephalic version, $N H D U=$ Neonatal high-dependency unit, $N I C U=$ Neonatal intensive care unit,$S C B U=S p e c i a l$ care baby unit, US = UItrasound. GMFCS = Gross Motor Function Classification System, ranging from 1 (mild) to 5 (severe).

All costs in pound sterling (f) and updated to the cost-year of 2016-17 using the HCHS Index ${ }^{56}$. 


\section{Derivation of input values for costs}

\section{Costs of ultrasound scan for foetal size}

The cost of an ultrasound scan was extracted from the national schedule of reference costs (Outpatient procedures, 'Ante-Natal Standard Ultrasound scan (NZ21Z)'). ${ }^{43}$ Weighted average mean and inter-quartile ranges for costs were calculated, and a gamma distribution fit to these. Resulting parameters: $\alpha=4.6904, \beta=22.8062$, yielding a mean of $f 107.06$ (95\% Cl: 70.89, 134.92).

\section{Cost of ultrasound scan for foetal presentation only}

There is no published unit cost for a presentation-only scan as it is not routinely undertaken in the NHS. Therefore, we costed two alternative scenarios:

\section{Midwife-led screening in primary care setting}

We hypothesised that a midwife could perform a scan as part of a standard antenatal visit in primary care, using a basic handheld scanner connected to a mobile phone or tablet computer (point of care ultrasound). Following the methodology for Wastlund et al. ${ }^{14}$, the cost for the presentation-only scan was estimated as a function of the midwife's time, equipment cost, and overheads (room/facilities).

The hourly cost for a Band 5 nurse in 2017 was $£ 36 .{ }^{56}$ The scan is assumed to take 5-10 minutes comprising time to make the mother comfortable, the scan itself and documentation of results. In the absence of data on the cost of ultrasound equipment and midwife training, we estimated a total cost of between $f 1,000-20,000$ and the average scanner is operated 400 to 3000 times annually over its 5-year life-span. Room costs are assumed between $£ 4,500$ and $£ 6,000$ annually ${ }^{58}$, and in use for the scans 1,573 hours per year. ${ }^{56}$

The total cost was simulated using uniform distributions 100,000 times, and a gamma distribution fitted to the resulting distribution. The resulting parameters of the gamma are alpha $=43.8259$, beta $=0.2159$, yielding a mean cost of $£ 9.46(95 \% \mathrm{Cl}: £ 6.87,12.46)$.

\section{Sonographer-led ultrasound in designated setting}

If the midwife-led scenario proves infeasible, the alternative is referral to a designated ultrasonography unit. A presentation-only scan is much swifter and technically less complicated than a standard antenatal scan. Reference cost 'Diagnostic imaging, Ultrasound Scan with duration of less than 20 minutes, without Contrast (RD40Z)' was used reporting mean ( $₫ 52$ ) and inter-quartile range ( $₫ 37-60)$, to which a gamma distribution was fitted (alpha $=9.2207$, beta $=5.6395)$, yielding a mean of $£ 52.00$ and $95 \% \mathrm{Cl}: £ 24.05, £ 90.55$.

\section{Cost for base-case scenario}

To incorporate uncertainty over the feasibility of a midwife-led presentation-only scan, we used a uniform distribution of costs, ranging between the lower end of the $95 \% \mathrm{Cl}$ for a midwife-led scan ( $f 6.87)$ and the upper end of the $\mathrm{Cl}$ for sonographer-led scan ( $₫ 90.55)$.

\section{Cost per mode of delivery}

For each of the three modes of deliveries (cephalic vaginal, planned CS and emergency CS), weighted averages of cost by admission type (in/out patient, elective/non-elective etc) and level of complications reported in NHS reference costs ${ }^{43}$ were calculated, to which a gamma distribution was fitted. For vaginal delivery, this yielded $\alpha=7.2606, \beta=252.5824$, with a mean of $£ 1,834.47$ (95\% Cl: $£ 1750.43,2236.05)$. For planned CS: $\alpha=11.1212, \beta=307.0169$, with a mean of $£ 3,411.93(95 \% \mathrm{Cl}$ : 
$£ 2679.80,4038.29)$. For emergency CS: $\alpha=14.7329, \beta=318.1354$, with a mean of $£ 4,688.27(95 \% \mathrm{Cl}$ : £3816.15, 5443.02)

NHS reference costs do not cost vaginal breech deliveries separately. We therefore assumed these costs would have the same ratio to the costs of elective caesarean section as reported by Palencia et al. (2006). ${ }^{45}$ The authors reported Ca\$7,255 and Ca\$8,440 for elective caesarean section and vaginal breech delivery, respectively, with a mean difference of Ca $\$ 1,185(95 \% \mathrm{Cl}: \$ 719, \$ 1663)$. We fitted a normal distribution to this ( mean $=1.1633$, $s d=0.0332$ ). The cost of vaginal breech delivery was calculated by multiplying the cost of elective $\mathrm{CS}^{43}$ with the relative cost increase from vaginal breech.

\section{Cost of External Cephalic Version (ECV)}

External cephalic version (ECV) cost was estimated from a 2001 UK based study. ${ }^{46} \mathrm{~A}$ low ( $\mathrm{f} 186.70$ ) and high (£193.30) staff cost scenario are reported by the authors. These were converted to 2017 prices using the Hospital \& Community Health Services (HCHS) inflation index, ${ }^{56} 57$ yielding $£ 287.20$ and f297.40 for low and high staff costs. These were assumed the minimum and maximum plausible costs and a uniform distribution assigned between them.

\section{Cost of neonatal unit admission}

Neonatal critical care was divided into three levels: 'Intensive care', 'High-dependency', and 'Special care'. Intensive and high dependency care were assigned currency codes XA01Z and XA02Z from the NHS reference costs. ${ }^{43}$ Special care was costed using a weighted average of currency codes XA03Z to XA05Z. ${ }^{43}$ Proportions of neonates admitted to each level of care and length of stay was extracted from Alfirevic et al.: ${ }^{47} 19 \%, 7 \%$, and $74 \%$ percent of admitted neonates went to intensive, high dependency, and special care, with a length of stay of 2, 1.5, and 2 days, respectively. Gamma distributions were fitted to the reported mean and IQRs from NHS reference costs.

Data from the POP study ${ }^{12}$ were reanalysed to estimate the probability of admission to neonatal care as a function of neonatal morbidity. Apgar score $(5 \mathrm{~min}$ ) was assumed a proxy for neonatal morbidity at delivery with score $>7,4-6$, and 0-3 were equivalent to no, moderate and severe neonatal morbidity. Based on POP study data, $7.4 \%$ (95\% Cl: 6.6-8.2\%) of neonates with no morbidity, and $47.4 \%(95 \% \mathrm{Cl}$ : 31.9-63.1\%) with moderate morbidity were admitted to care. Beta distributions were fitted to these proportions. Sample sizes of neonates with severe morbidity were too small to reliably estimate proportions admitted. We therefore assumed all neonates with severe morbidity would be admitted to care. In absence of evidence of how the level of neonatal morbidity at birth affects the chance of ending up in each tier of neonatal care, we assumed that the proportions were constant, and that the level of neonatal morbidity only affected the level of overall admittance.

\section{Cost from Respiratory morbidity}

A 1995 study ${ }^{27}$ of the incidence and length of stay at hospital for respiratory morbidity in neonates found 28\% were for Respiratory Distress Syndrome (RDS) and the rest of Transient Tachypnea of the Newborn (TTN). Average length of stay in Neonatal Intensive Care Unit (NICU) was 4 days for RDS and 0.6 days for TTN. The NHS reference cost of NICU admission is $£ 1,295$ per day (IQR: $£ 1,015-1,541) .{ }^{43}$ Thus the average cost for a case of RDS is $f 5,180$ (IQR: $£ 4,060-6,164$ ), and for $T T N, f 777$ (IQR: 609925). If RDS and TTN comprise $28 \%$ and $72 \%$ of respiratory morbidities respectively, the mean cost of respiratory morbidity is $f 2,010$ (IQR: $f 1,575-2,392$ ). Due to the very low mortality rate from respiratory distress among babies born at term, we assumed respiratory distress could lead to NICU admission, but would otherwise have no consequences. ${ }^{59} \mathrm{~A}$ gamma distribution was fitted to these data, yielding alpha $=10.7125$, beta $=187.6316$, and a mean of $£ 2011(95 \% \mathrm{Cl}: £ 993, £ 3381)$. 


\section{Cost of acidosis without long-term consequences}

In the absence of data, we assumed acidosis led to admission to neonatal intensive care unit (NICU) for 1-4 days, with equal probabilities. A gamma distribution was fitted to per-diem costs from NHS reference costs ${ }^{43}$. A gamma distribution was fitted to combined length of stay and per diem cost (alpha $=3.6143$, beta $=895.6169)$ yielding a mean of $£ 3,240(95 \% \mathrm{Cl}$ : $£ 806-7,328)$.

\section{Cost of transient and permanent BPI}

Brachial Plexus Injury (BPI) costs were based on Culligan et al. (2010) ${ }^{48}$. Transient BPI resource use comprised specialist hospital consultation, weekly physical therapy for 4 months, and one needle electromyography (EMG) test. Permanent BPI resource use was assumed the same as transient, but with weekly physical therapy for 3 years rather than 4 months, plus one outpatient visit to a specialist, and magnetic resonance imaging (MRI) of the shoulder. ${ }^{48}$ Cost for specialist consultations and weekly physiotherapy treatments were $£ 199$ and $£ 87$, respectively. ${ }^{60} \mathrm{EMG}$ and MRI costs were $£ 269.20$ and f106.59 respectively (NHS reference costs, codes AA33D and RD01C). ${ }^{43}$ All costs were adjusted to 2016-17 prices using the HCHS index..$^{56}$ We assumed that all costs except for physiotherapy arose in the first year of life and discounted subsequent costs at $3.5 \% .{ }^{64}$ The total discounted costs from transient and permanent BPI were $£ 2,066$ and $£ 14,133$, respectively.

Culligan et al. ${ }^{48}$ assumed costs would vary between $50-200 \%$ of their point estimate. Directly incorporating this into our model (after adjusting for cost differences) with a uniform distribution was considered inappropriate as it would substantially overestimate costs. We therefore interpreted the plausible range as a $95 \%$ confidence interval $(\mathrm{Cl})$ for total costs, fitting a log-normal distribution to the appropriate mean and $\mathrm{Cl}$ range. For transient $\mathrm{BPI}$, the resulting distribution had a logged standard error of 0.3536 , and mean cost $£ 2,066$ (95\% Cl: $£ 1033-4132)$. For permanent BPI the logged standard error was 0.3536 , with a mean of $£ 14,133$ (95\% Cl: $f 7067-28264)$.

\section{Cost of perinatal death}

The cost of stillbirth was assumed a proxy for the cost of perinatal death. Mistry et al. ${ }^{49}$ estimated a cost of between $£ 1,242$ (core investigation and counselling only) and $£ 1,804$ depending on the clinical scenario surrounding the stillbirth and what tests were needed. We adjusted these estimates to 201617 prices ${ }^{56}$, and assigned a uniform distribution between them.

\section{Cost of special educational needs (SEN)}

Barrett et al. ${ }^{50}$ estimated an additional cost of SEN of $£ 6,315(95 \% \mathrm{Cl}$ : $£ 3798,8832)$ per annum in 200708 prices. Inflated to $2016-17$ prices $^{56}$ resulted in an additional cost of $£ 7,428$ ( $95 \% \mathrm{Cl}$ : $£ 4467,10389$ ). This was applied annually for years 6-17 of life (the typical school years) and discounted at $3.5 \%{ }^{64}$

\section{The cost of severe neurological morbidity}

Cerebral palsy (CP) was assumed a proxy for severe neurological morbidity. In the absence of relevant UK data, annual cost was based on Australian data ${ }^{51}$. We extracted total per capita cost for the health system, including program services, aids, and home modifications, but omitted productivity losses, dead weight losses from financial transactions, and costs for informal carers. The annual average cost in 2005 was 5,362 AUD. Adjusted to GPB using the exchange rate at $31^{\text {st }}$ Dec 2005 and inflated to $2016 / 17$ prices $^{56}$ yielded an annual mean cost of $£ 2,929.60$. In the absence of relevant data, we assumed a $95 \% \mathrm{Cl}$ around the mean at $50 \%$ and $200 \%$ of the mean $(£ 1465, f 5859)$, to which a lognormal distribution was fitted. 


\section{Derivation of input values for QALYS}

\section{Baseline long-term Quality-Adjusted Life Years}

Lifetime quality-adjusted life years (QALYs) were calculated using survival and Quality of Life (QoL) weights for the general UK population, ${ }^{5361}$ discounted at $3.5 \%{ }^{64}$ Stillbirth was assumed to accrue zero QALYS.

\section{Quality of life for brachial plexus injury (BPI)}

Culligan et al. ${ }^{48}$ used an expert panel to estimate a plausible range of health state utilities for BPI by severity, to which was assigned a uniform distribution. Our model definition of long-term BPI was assumed equivalent to Culligan et al.'s state of either 'Permanent brachial plexus injury (mild to moderate)', or 'Permanent brachial plexus injury (severe) and uncomplicated delivery'. This yielded a uniform distribution between 0.30 (the lower boundary for severe BPI) and 0.70 (the upper boundary for mild to moderate $\mathrm{BPI})$.

\section{Long-term health outcomes following severe neurological morbidity}

We assumed cerebral palsy was a proxy for all severe neurological morbidity. Analogous to Leigh et al., ${ }^{54}$ we divided cerebral palsy into the five levels of the Gross Motor Function Classification System (GMFCS), which focuses on ambulatory functionality of people with CP. ${ }^{62}$ GMFCS-specific quality of life (QoL) was assigned a gamma distribution from values provided by Leigh et al. ${ }^{54}$, subtracting these values from 1 (highest possible QoL) to provide utility weights. QoL was assumed to decrease over time at the same rate as Leigh et al. This effectively assumes that ageing has no greater effect on QoL for those with CP than otherwise healthy members of the UK.

GMFCS-specific survival rates were extracted from Leigh et al. by age band (0-10 years, 11-20 years, and 21-30 years). In the absence of evidence on GMFCS-specific mortality rates beyond 30 years of life, we made the conservative assumption that the mortality rate for those born with severe neurological morbidity who had survived to age 30 would mimic the general population in the UK after this age.

Young et al..$^{52}$ report the distribution of GMFCS states to which we assigned a Dirichlet distribution.

Combining QoL with survival, and the distribution of GMFCS states, we obtained expected lifetime QALYs accrued for neonates born with severe neurological morbidity. QALYs accrued after year 1 were discounted at $3.5 \%{ }^{64}$

\section{Beneficial population}

Value of information analyses require an estimate of the population who can benefit from the information yielded from research. The target population is all singleton births to nulliparous women in England, excluding those opting for elective CS for reasons other than breech presentation.

There were 636,401 births in England in FY2016-17. ${ }^{63}$ Of these, $91.8 \%$ were at $\geq 37$ weeks' gestational age, out of which $33.6 \%$ were to nulliparous mothers. ${ }^{63}$ The statistics do not disaggregate by reason for elective CS (specifically, whether because of suspected breech position or not). Therefore, this means there were:

$$
636,401 * 0.918 * 0.336=196,297
$$


deliveries in England annually meeting our population definition.

Assuming a 10 year time horizon for the value of information analysis (a proxy for the length of time for which the decision question remains relevant before technological development changes it), no meaningful change in the number of deliveries per annum over that period and a discount rate of $3.5 \%$ yields a beneficial population of 1,689,663.

If our analyses are assumed generalisable to all pregnancies, then the beneficial population is 636,401 per annum, or 5,477,940 over the 10-year horizon (discounted at 3.5\%). 


\section{Appendix 3: EVI code walk-through}

Below is the code used to calculate the EVSI, with explanation in the righthand column. Full model code available on request from the corresponding author.

\begin{tabular}{|c|c|}
\hline Code & Comments / Walkthrough \\
\hline $\begin{array}{l}\text { EVI <- function(distributions=list }(c . \mid \mathrm{IOL}=c(" N ", 125.3,794.6,650)) \text {, } \\
n=100000, \text { lambda=20000, screens = screening, mgt = management, } \\
\text { popn }=636401, \text { Volhorizon=10, discountrate }=0.035, \\
Q=30, \text { samplesizes }=c(10,100,100000) \text {, paramSet = params })\{\end{array}$ & $\begin{array}{l}\text { Function header including some default values. } \\
\text { The default parameter of interest is called } \\
\text { 'c.IOL' (cost of induction of labour). It has a } \\
\text { normal distribution, with mean, standard error } \\
\text { and prior sample size of } 125.3,794.6 \text { and } 650 \text {. }\end{array}$ \\
\hline $\begin{array}{l}\text { \#Based on Heath \& Baio ViH } 2018 \\
\text { \# Note code is for } L N \text { and N priors written only. }\end{array}$ & \\
\hline $\begin{array}{l}\text { \# parameter - parameter name (must be same as column name in samp matrix) } \\
\text { \# n - number of PSA loops for calculating NB, EVPI and EVPPI } \\
\text { \# lambda - WTP threshold } \\
\text { \# screens \& mgt - different strategies } \\
\text { \# popn - beneficial population (per year) } \\
\text { \# horizon - time horizon over which to calculate EVI } \\
\text { \# discount rate - for discounting future values of EVI } \\
\text { \# Q - number of samples from prior distribution } \\
\text { \# samplesizes - vector of samplesizes of proposed study }\end{array}$ & $\begin{array}{l}\text { Comments describing input parameters for } \\
\text { function }\end{array}$ \\
\hline $\begin{array}{l}\text { parameters=names(distributions) } \\
\text { print(paste("calculating expected net benefits with current information using",n,"simulations...")) } \\
\text { set.seed(seed) } \\
\text { inputs <- samples(n,paramSet) } \\
\text { CNeoMorb <- costNeonatalMorb(inputs) \# calculates neonatal morbidity costs } \\
\text { LTCQ <- longtermCQ(discountRate, horizon, survival,inputs) \#calculates pv of long term costs and } \\
\text { QALYs } \\
\text { inputs <- cbind(inputs,CNeoMorb,LTCQ) \#bind LT costs and QALYs to end of inputs dataframe } \\
\text { inputs <- checkInputsAndCalculateCompoundProbs(inputs) \#adds in set of compound probabilities } \\
\text { (i.e. } P(y) \text { where } P(y)=P(x)^{*} R R \text { ) and checks for (and solves) out of bounds samples }\end{array}$ & $\begin{array}{l}\text { Run a standard PSA analysis of the model by } \\
\text { sampling all the inputs, ultimately storing the } \\
\text { output as a list item called } x \text {. }\end{array}$ \\
\hline
\end{tabular}




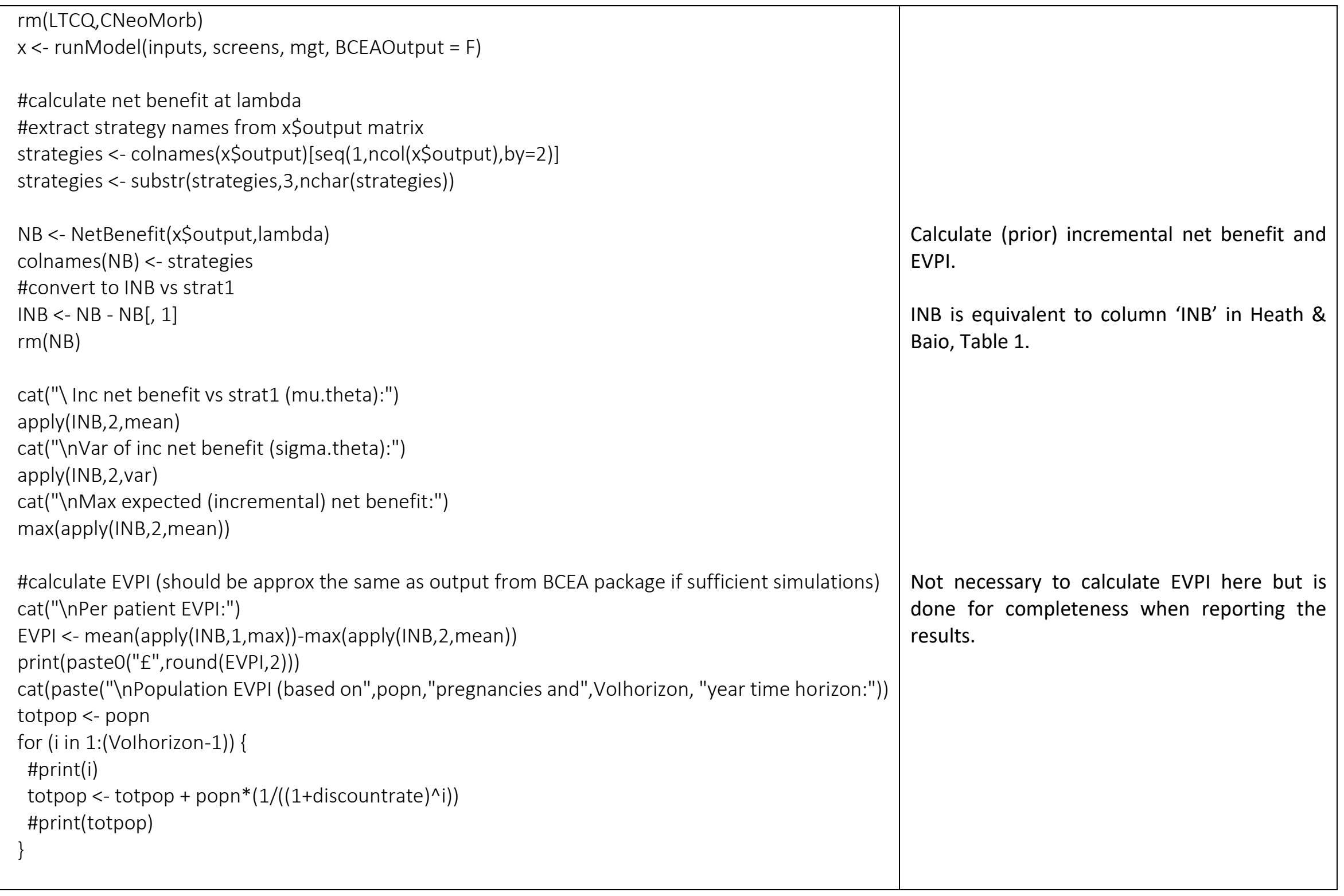




\begin{tabular}{|c|c|}
\hline $\begin{array}{l}\text { pEVPI = EVPI*totpop } \\
\text { print(pasteO("£",round(pEVPI,0))) } \\
\text { mu.theta <- apply(INB,2,mean) } \\
\text { sigma.theta <- apply(INB,2,var) }\end{array}$ & $\begin{array}{l}\text { Record prior INB and var(INB) for each strategy } \\
\text { (INB is all vs strategy } 1 \text { ) in two vectors, } \\
\text { mu.theta and sigma.theta. } \\
\text { These are equivalent to }-4.5 \text { and } 722 \text {, final two } \\
\text { rows of column 'INB' in Table } 1 \text {, Heath \& Baio. }\end{array}$ \\
\hline 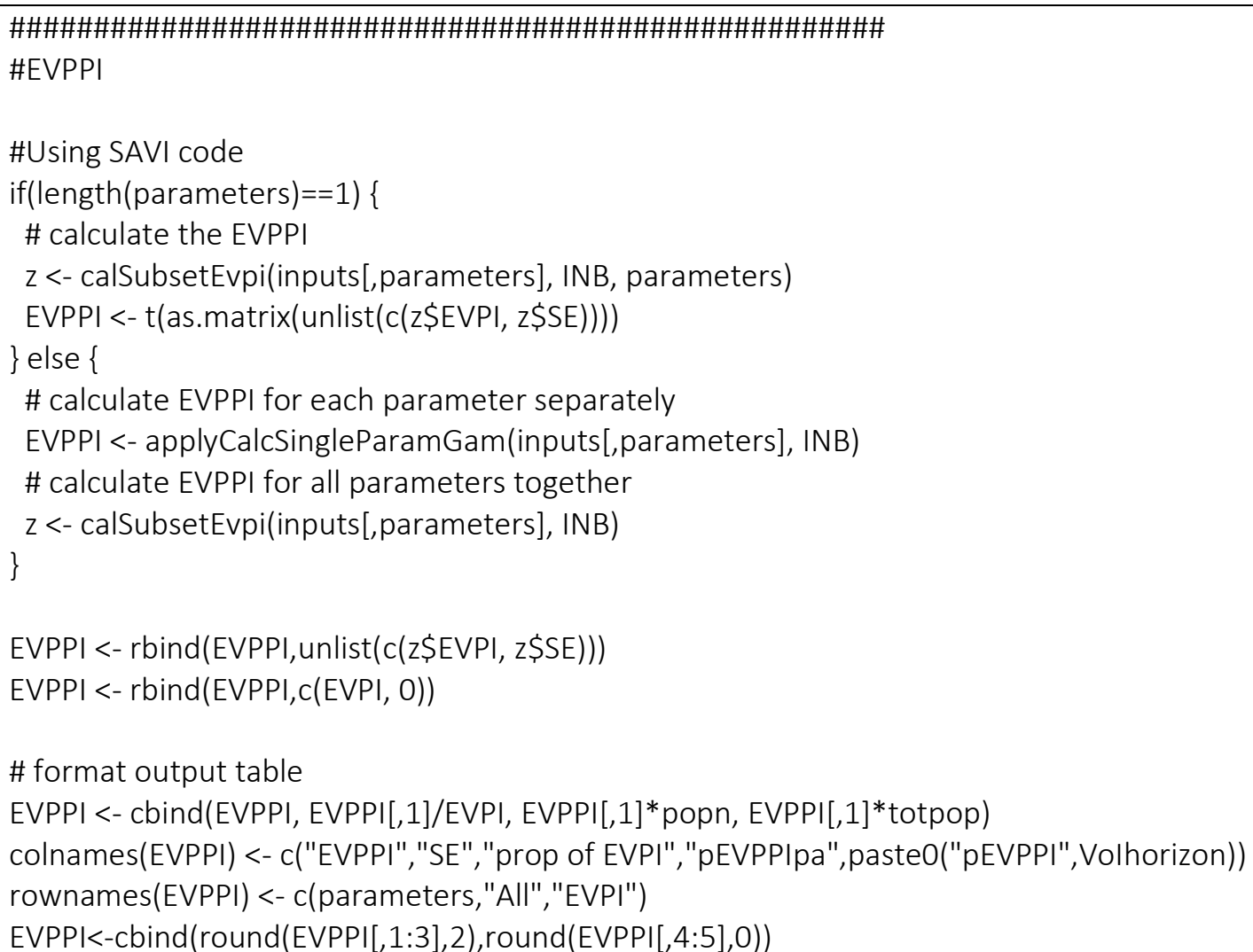 & $\begin{array}{l}\text { Calculate EVPPI for parameters of interest. } \\
\text { Functions here are SAVI functions, downloaded } \\
\text { from } \\
\text { Accelerated-Vol/SAVI. Code was modified to } \\
\text { return g.hat from the functions rather than } \\
\text { deleting it. } \\
\text { Output of calSubsetEvpi() is stored as a list } \\
\text { called z. }\end{array}$ \\
\hline
\end{tabular}




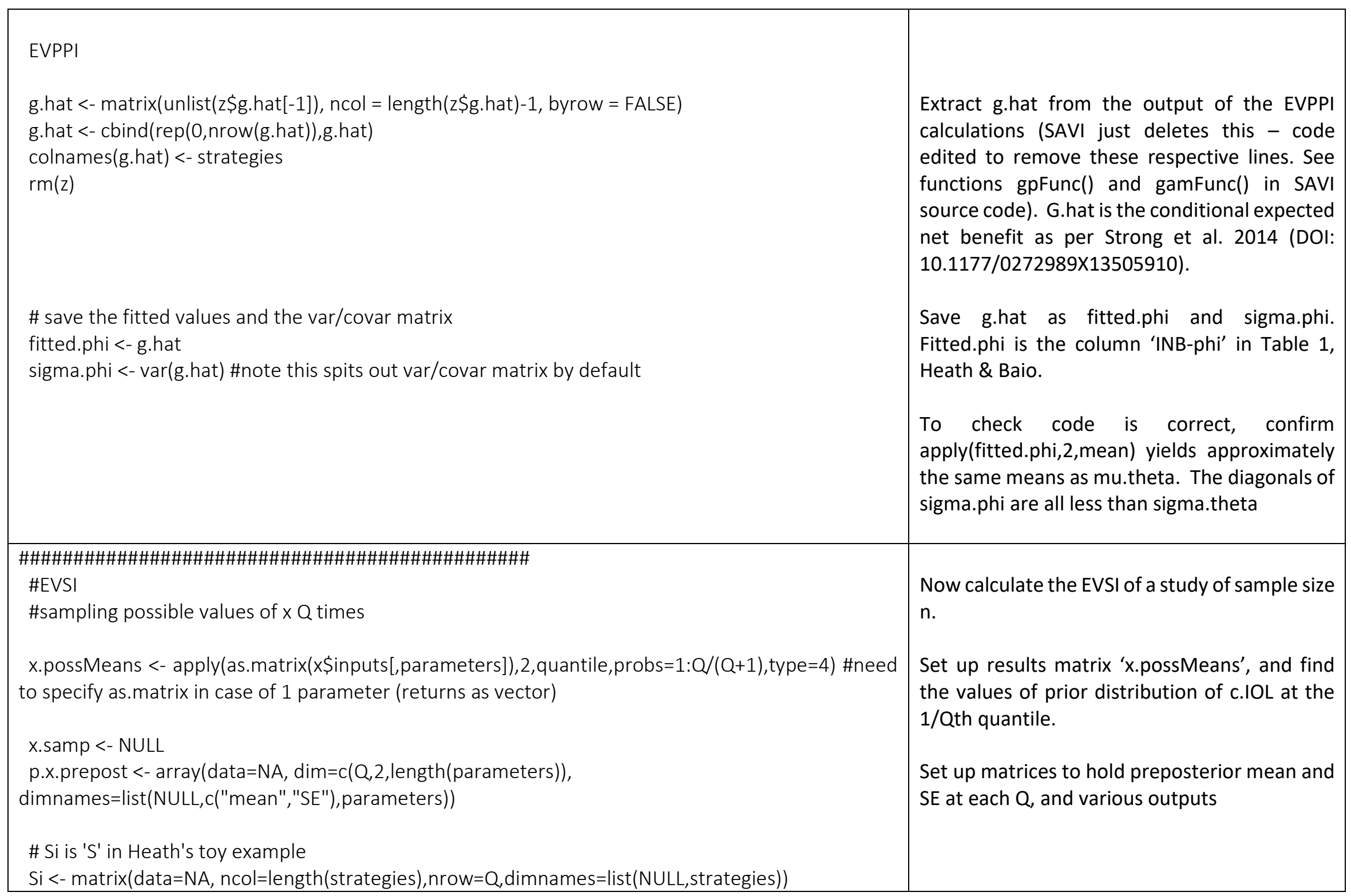




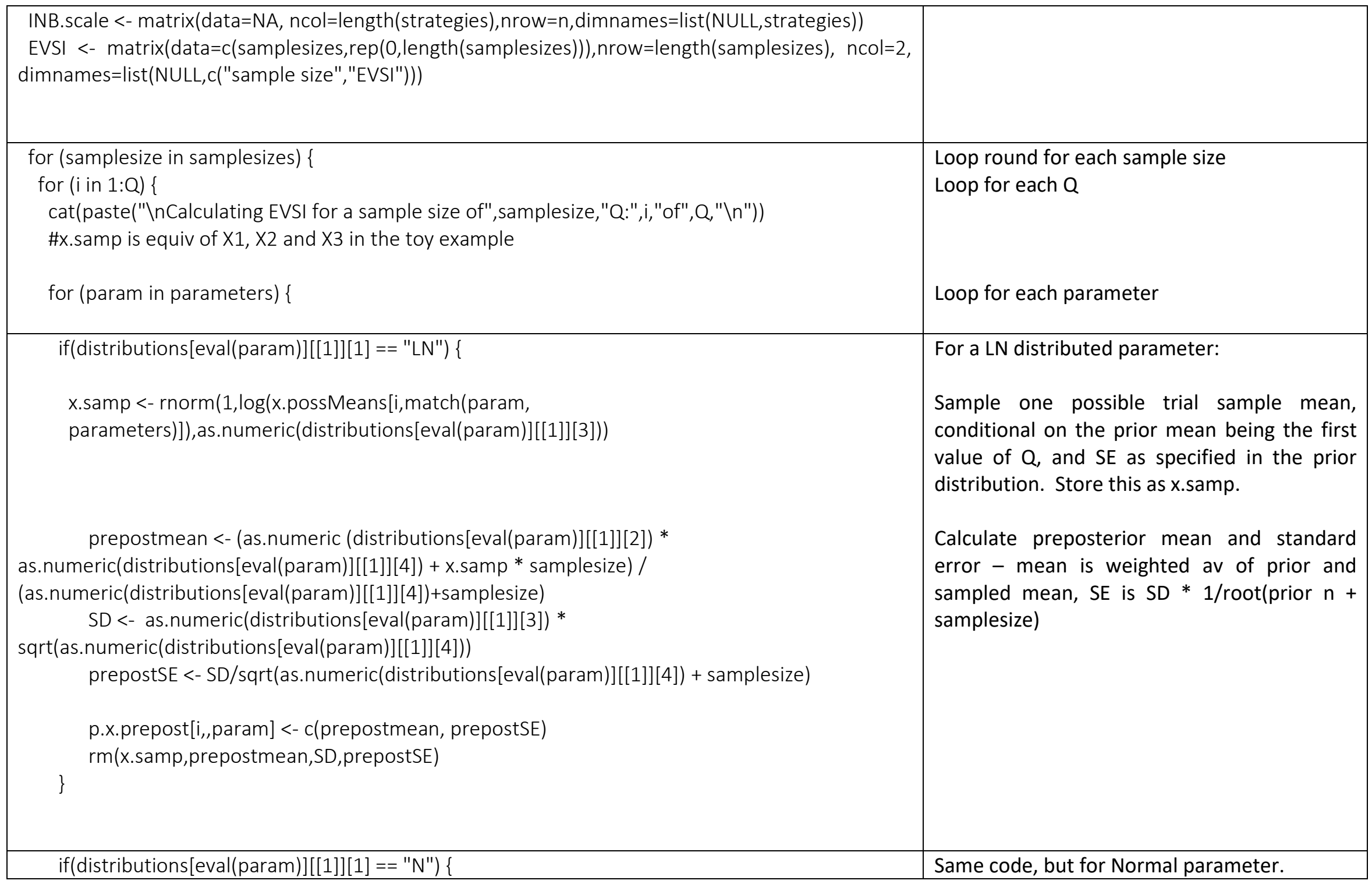




\begin{tabular}{|c|c|}
\hline $\begin{array}{l}\text { x.samp <- rnorm(1,x.possMeans[i,match(param, } \\
\text { parameters)],as.numeric(distributions[eval(param)][[1]][3])) } \\
\text { prepostmean <- } \\
\text { (as.numeric(distributions[eval(param)][[1]][2])*as.numeric(distributions[eval(param)][[1]][4])+x.sam } \\
\text { p*samplesize)/(as.numeric(distributions[eval(param)][[1]][4])+samplesize) } \\
\quad \text { SD <- } \\
\text { as.numeric(distributions[eval(param)][[1]][3])*sqrt(as.numeric(distributions[eval(param)][[1]][4])) } \\
\quad \text { prepostSE <- SD/sqrt(as.numeric(distributions[eval(param)][[1]][4])+samplesize) } \\
\text { p.x.prepost[i,,param]<- c(prepostmean, prepostSE) } \\
\text { rm(x.samp,prepostmean,SD,prepostSE) }\end{array}$ & \\
\hline$\}$ & Loop is repeated for every parameter \\
\hline $\begin{array}{l}\text { \#now calculate INB.X1, INB.X2 and INB.X3 (preposterior INB) } \\
\text { \# (calls samples() to generate inputs then replaces target parameters with preposterior samples) } \\
\text { samp <- samples(n, paramSet) } \\
\text { CNeoMorb <- costNeonatalMorb(samp) \# calculates neonatal morbidity costs } \\
\text { LTCQ <- longtermCQ(discountRate, horizon, survival,samp) \#calculates pv of long term costs and } \\
\text { QALYS } \\
\text { samp <- cbind(samp,CNeoMorb,LTCQ) \#bind LT costs and QALYs to end of inputs dataframe } \\
\text { samp <- checkInputsAndCalculateCompoundProbs(samp) \#adds in set of compound probabilities } \\
\text { (i.e. } P(y) \text { where } P(y)=P(x)^{*} \text { RR) and checks for (and solves) out of bounds samples }\end{array}$ & $\begin{array}{l}\text { Calculation of preposterior INB } \\
\text { Sample from the prior distribution of every } \\
\text { parameter }\end{array}$ \\
\hline $\begin{array}{l}\text { for (param in parameters) }\{ \\
\text { if(distributions[eval(param)][[1]][1] == "LN") }\{ \\
\text { samp[,param] <- exp(rnorm(n, p.x.prepost[i,1,param], p.x.prepost[i,2,param])) } \\
\} \\
\text { if(distributions[eval(param)][[1]][1] == "N") }\{ \\
\text { samp[,param] <- rnorm(n, p.x.prepost[i,1,param], p.x.prepost[i,2,param]) }\end{array}$ & $\begin{array}{l}\text { Replace sampled values of parameters of } \\
\text { interest with samples from the pre-posterior } \\
\text { distributions. } \\
\text { (Note it was more expedient to code the model } \\
\text { to sample all parameters using the prior }\end{array}$ \\
\hline
\end{tabular}




\begin{tabular}{|c|c|}
\hline$\}$ & $\begin{array}{l}\text { distributions, then replace with the } \\
\text { preposteriors rather than changing the } \\
\text { distributions sent to the samples() function) }\end{array}$ \\
\hline $\begin{array}{l}\text { output }<- \text { runModel(samp, screens, mgt, BCEAOutput = F)\$output } \\
\text { cat(paste("Calculating preposterior NB with",n," loops. }(n ")) \\
\text { NB }<- \text { NetBenefit(output,lambda) } \\
\text { colnames(NB) <- strategies } \\
\text { \#convert to INB rel to strat } 1 \text { (as used in SAVI code) } \\
\text { INB <- NB - NB[, 1] }\end{array}$ & $\begin{array}{l}\text { Run the model with the sampled PSA values } \\
\text { and calculate preposterior INB }\end{array}$ \\
\hline $\begin{array}{l}\text { cat("storing variances of preposterior net benefit } \backslash n ") \\
\text { Si[i,] <- apply(INB,2,var) }\end{array}$ & $\begin{array}{l}\text { Save preposterior variance of INB for each } \\
\text { strategy }\end{array}$ \\
\hline$\}$ & Repeat process for all Q \\
\hline sigma.X $<-$ apply(Si,2,mean) & $\begin{array}{l}\text { Calculate mean preposterior variance of INB } \\
\text { for each strategy across all } Q \text {. }\end{array}$ \\
\hline $\begin{array}{l}\text { INB.scale[,1] }=0 \\
\text { for }(\mathrm{i} \text { in } 2: \text { ncol(Si)) }\{ \\
\quad \text { INB.scale[,i] }<-\quad(\text { fitted.phi[,i]-mu.theta[i])/sqrt(sigma.phi[i,i])*sqrt(max }(0, \text { sigma.theta[i]- } \\
\text { sigma.X[i]))+ mu.theta[i] } \\
\quad\}\end{array}$ & $\begin{array}{l}\text { Rescaled INB as per Heath \& Baio. } \\
\text { Note the requirement for the } \\
\text { max(0,sigma.theta[i]-sigma.X[i]). For some } \\
\text { parameters, the estimated preposterior } \\
\text { variance is greater than prior. This occurs } \\
\text { where the prior for, eg a probability between } \\
\text { two long term outcomes is very vague and one } \\
\text { of the outcomes is very certain and the other is } \\
\text { highly uncertain (eg death vs a highly uncertain } \\
\text { future prognosis). Methodological work } \\
\text { exploring this is ongoing. }\end{array}$ \\
\hline $\begin{array}{l}\text { \#EVSI } \\
\text { EVSI[match(samplesize,EVSI),2] <- mean(apply(INB.scale,1, max))-max(mu.theta) }\end{array}$ & Calculate EVSI \\
\hline$\}$ & Repeat for every sample size \\
\hline
\end{tabular}




\begin{tabular}{|l|l|}
\hline EVSI <- cbind(EVSI,EVSI[,2]*popn,EVSI[,2]*totpop) & Tidy up EVSI results table and return EVPPI and \\
colnames(EVSI)[3:4] =c("pEVSIpa","pEVSI10") & EVSI \\
\# EVSI[,2]<-round(EVSI[,2],4) \\
EVSI[,3:4] <-round(EVSI[,3:4],0) \\
$\begin{array}{l}\text { list(EVPPI = EVPPI, } \\
\text { EVSI = EVSI) }\end{array}$ \\
\}$\quad$ \\
\hline
\end{tabular}




\section{Appendix 4: Stability testing}

Stability testing was conducted to quantify (and thence minimise) Monte Carlo error as a function of the number of simulations. The model was run 30 times with a given number of simulations. The coefficient of variation of the estimates of mean and standard error of mean cost and QALYs for each comparator were calculated. The mean of all of these was used as a summary measure of the Monte Carlo error. We used an arbitrary $2 \%$ cut-off to declare the results stable.

Our analyses showed that we were able to achieve extremely stable results (coefficient of variation of $<0.01 \%$ ) with 100,000 simulations, at a 'reasonable' run time of around 30 seconds. We therefore run our cost-effectiveness analyses with 100,000 simulations. However, due to the need for repeated loops, the EVSI calculations are based on 10,000 simulations. This still generates stable results with a coefficient of variation of only $0.56 \%$.

Figure A3.1. Stability testing

\begin{tabular}{ccc} 
Simulations & $\begin{array}{c}\text { Computation time } \\
\text { (seconds) }\end{array}$ & $\begin{array}{c}\text { Mean Coefficient of Variation } \\
(\%)\end{array}$ \\
\hline 10 & 0.10 & 24.68 \\
100 & 0.09 & 7.73 \\
1000 & 0.33 & 2.53 \\
10000 & 2.75 & 0.56 \\
100000 & 29.56 & $<0.01$
\end{tabular}




\section{Appendix 5: One-way sensitivity analyses}

The following figures show the relationship between the parameter of interest and (expected) net benefit of each strategy (net benefit shown as incremental net benefit compared with strategy 1 , assumed to represent status quo). The option with the highest net benefit (or equivalently, the highest incremental net benefit versus strategy 1 ) is the most cost-effective, on average. Net benefit calculated at $£ 20,000$ per QALY gained.

Figure A4.1. One-way sensitivity analysis on model time horizon, showing expected incremental net benefit relative to status quo.

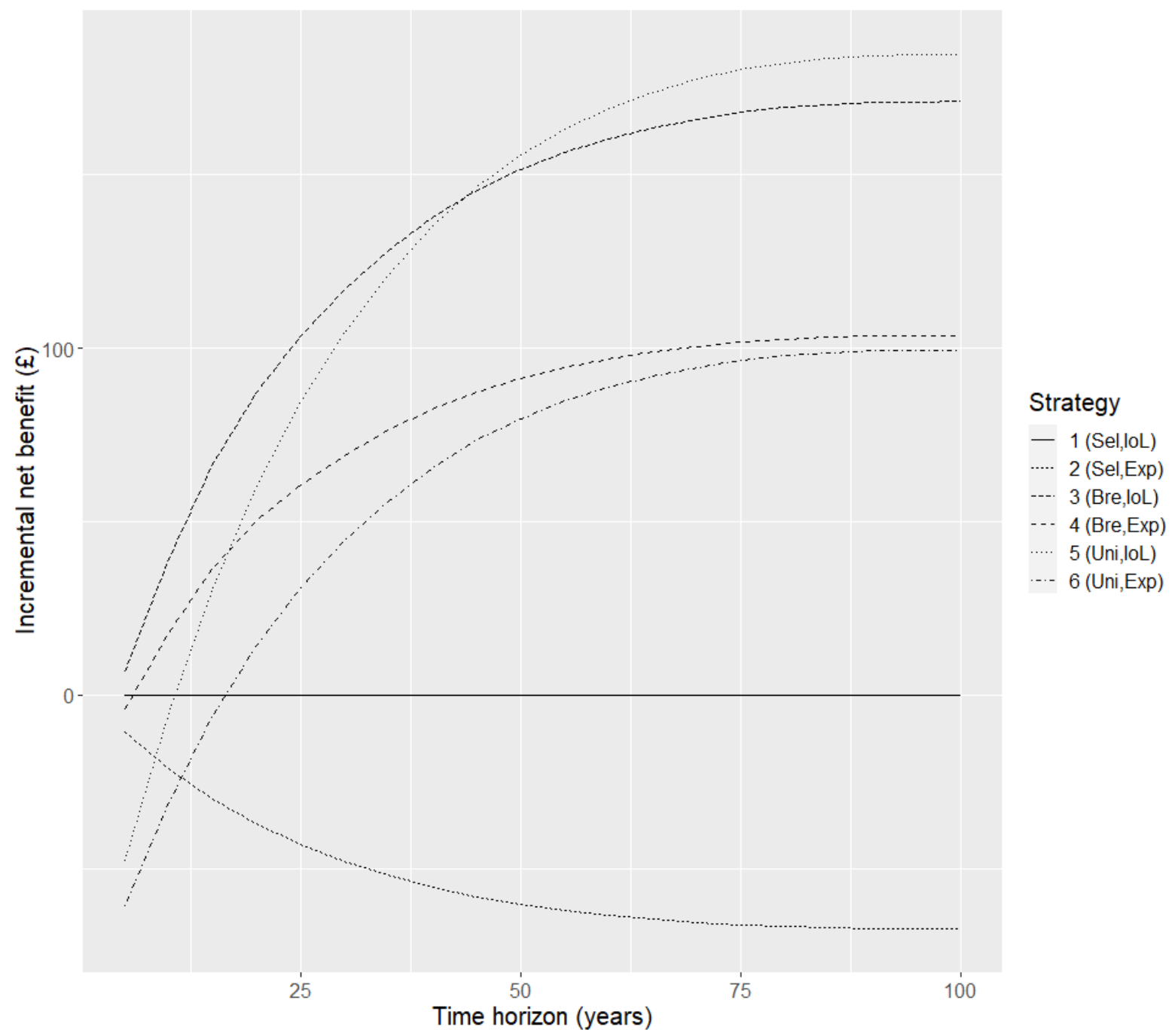

Sel = selective scanning; Bre = Universal presentation-only scan; Uni = Universal scan of foetal biometry and presentation; $I O L=$ Induction of labour if $L G A$ suspected; $\operatorname{Exp}=$ Expectant management if $L G A$ suspected. 
Figure A4.2. One-way sensitivity analysis on the cost of a scan for foetal presentation only, showing expected incremental net benefit relative to status quo.

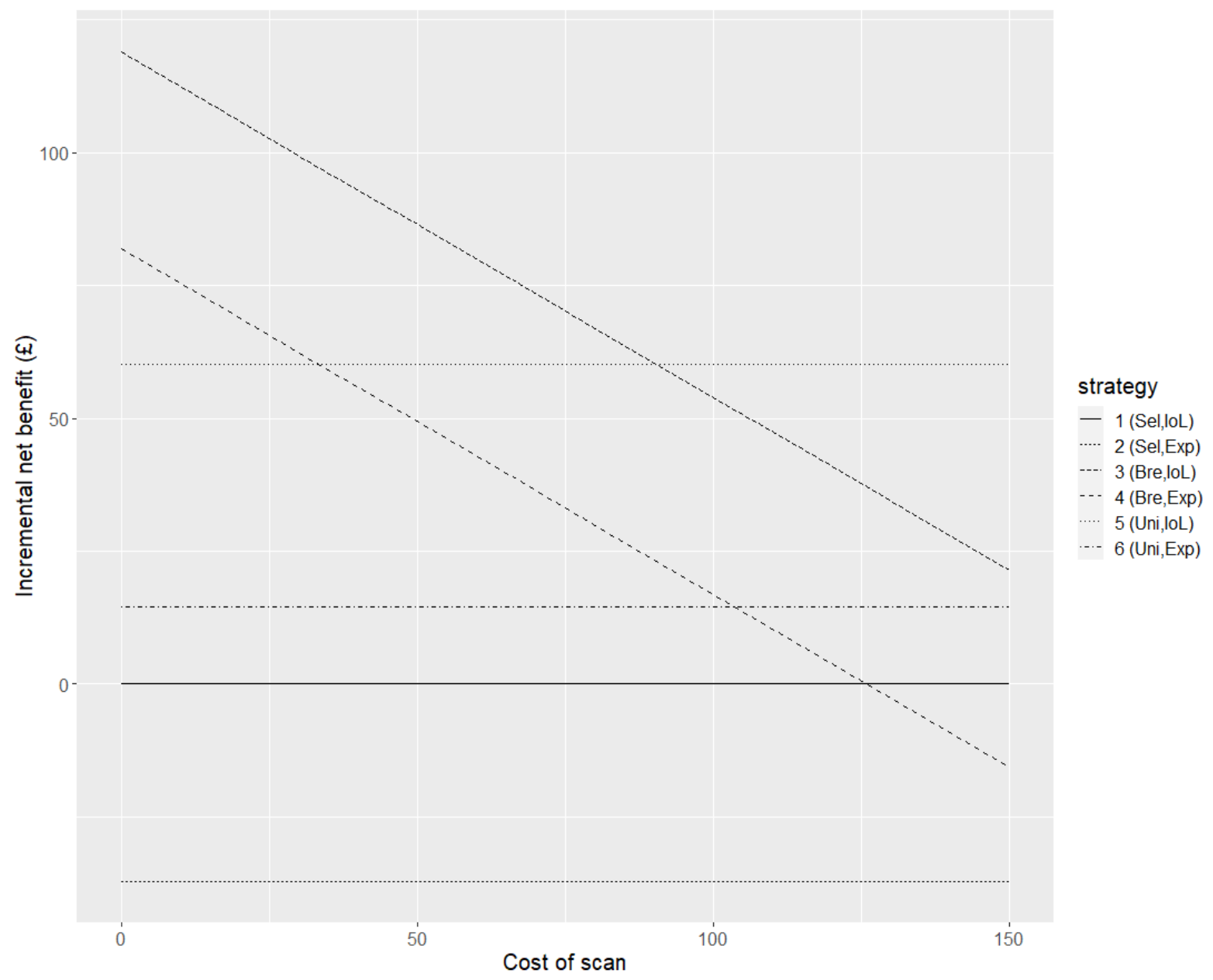

Sel = selective scanning; Bre = Universal presentation-only scan; Uni = Universal scan of foetal biometry and presentation; $I o L=$ Induction of labour if $L G A$ suspected; Exp = Expectant management if $L G A$ suspected. 
Figure A4.3a, A4.3b and A4.3c. One-way sensitivity analysis on baseline risk of perinatal mortality, moderate and severe morbidity respectively, showing expected incremental net benefit relative to status quo.
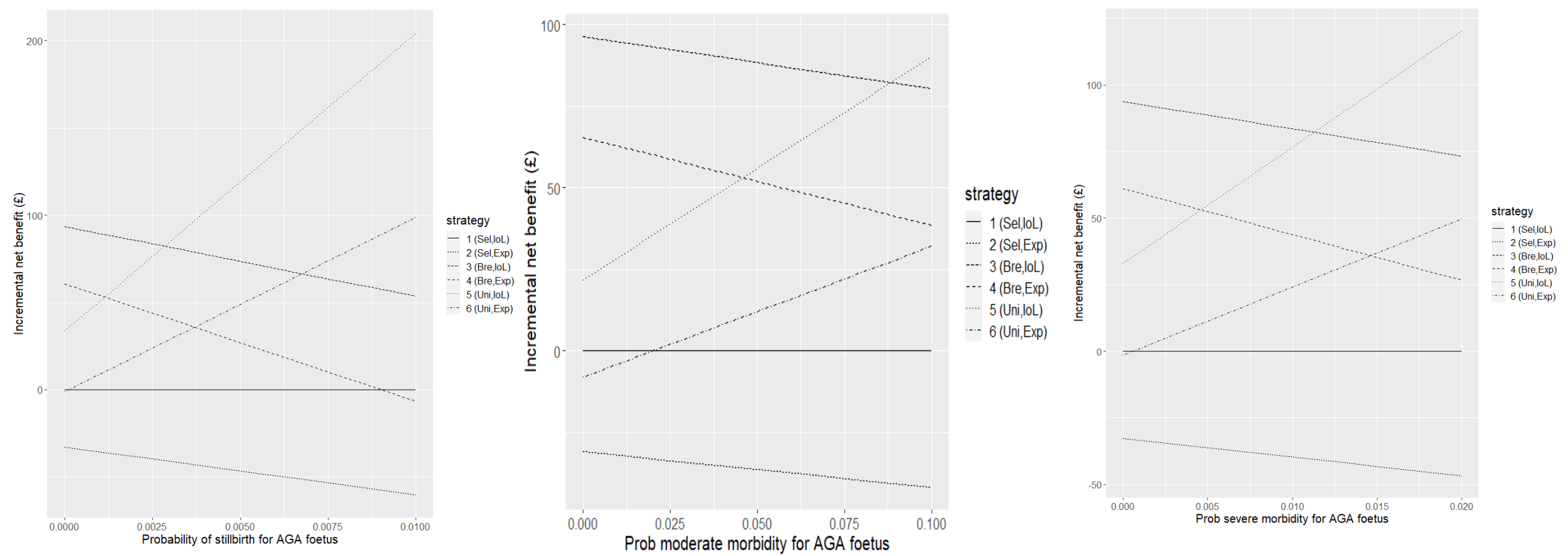

Sel = selective scanning; Bre = Universal presentation-only scan; Uni = Universal scan of foetal biometry and presentation; IoL = Induction of labour if LGA suspected; Exp = Expectant management if LGA suspected. $A G A=$ average size for gestational age (i.e. not SGA or LGA) 
Figure A4.4. One-way sensitivity analysis on the relative risk of special educational needs following induction of labour, showing expected incremental net benefit relative to status quo.

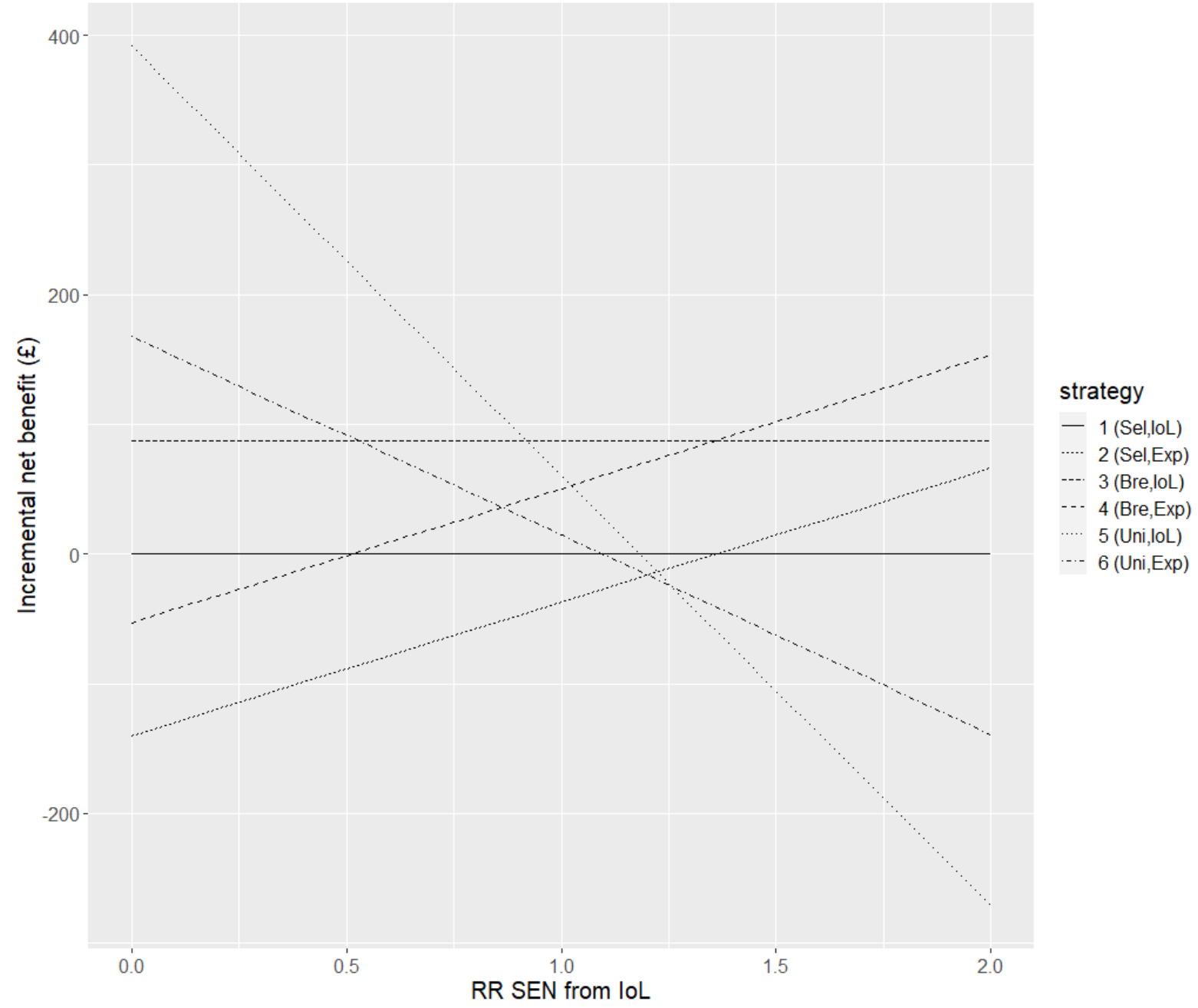

Sel = selective scanning; Bre = Universal presentation-only scan; Uni = Universal scan of foetal biometry and presentation; $I O L=$ Induction of labour if $L G A$ suspected; Exp = Expectant management if $L G A$ suspected; SEN = Special Educational Needs; $R R=$ relative risk . 